\title{
The VLT-FLAMES survey of massive stars: Observations in the Galactic clusters NGC 3293, NGC 4755 and NGC $6611^{\star, \star \star}$
}

\author{
C. J. Evans ${ }^{1}$, S. J. Smartt ${ }^{2}$, J.-K. Lee ${ }^{2}$, D. J. Lennon ${ }^{1}$, A. Kaufer ${ }^{3}$, P. L. Dufton ${ }^{2}$, C. Trundle ${ }^{4}$, A. Herrero ${ }^{4,5}$, \\ S. Simón-Díaz ${ }^{4}$, A. de Koter ${ }^{6}$, W.-R. Hamann ${ }^{7}$, M. A. Hendry ${ }^{8}$, I. Hunter ${ }^{2}$, M. J. Irwin ${ }^{8}$, A. J. Korn ${ }^{9}$, \\ R.-P. Kudritzki ${ }^{10}$, N. Langer ${ }^{11}$, M. R. Mokiem ${ }^{6}$, F. Najarro ${ }^{12}$, A. W. A. Pauldrach ${ }^{13}$, N. Przybilla ${ }^{14}$, J. Puls ${ }^{13}$, \\ R. S. I. Ryans ${ }^{2}$, M. A. Urbaneja ${ }^{10}$, K. A. Venn ${ }^{15}$, and M. R. Villamariz ${ }^{4}$
}

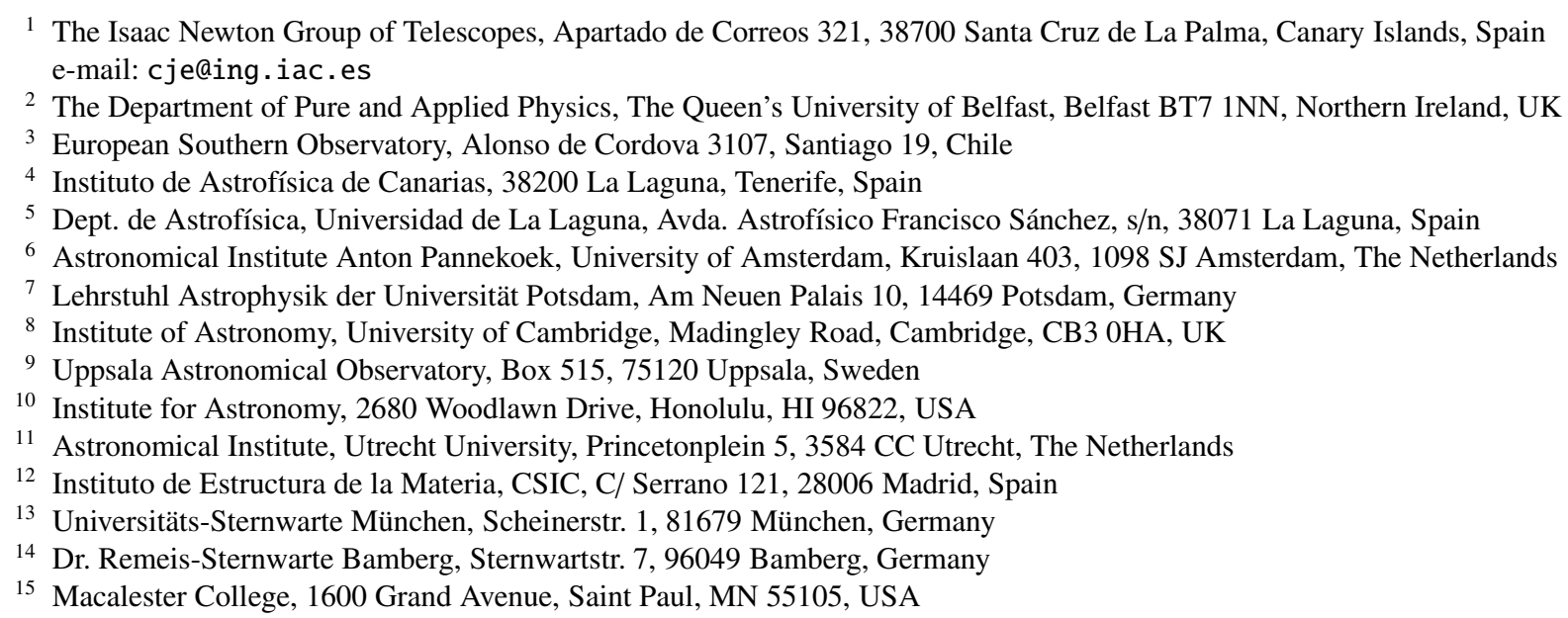

Received 29 November 2004 / Accepted 14 March 2005

\begin{abstract}
We introduce a new survey of massive stars in the Galaxy and the Magellanic Clouds using the Fibre Large Array Multi-Element Spectrograph (FLAMES) instrument at the Very Large Telescope (VLT). Here we present observations of 269 Galactic stars with the FLAMES-Giraffe Spectrograph $(R \simeq 25000)$, in fields centered on the open clusters NGC 3293, NGC 4755 and NGC 6611. These data are supplemented by a further 50 targets observed with the Fibre-Fed Extended Range Optical Spectrograph (FEROS, $R=48000$ ). Following a description of our scientific motivations and target selection criteria, the data reduction methods are described; of critical importance the FLAMES reduction pipeline is found to yield spectra that are in excellent agreement with less automated methods. Spectral classifications and radial velocity measurements are presented for each star, with particular attention paid to morphological peculiarities and evidence of binarity. These observations represent a significant increase in the known spectral content of NGC 3293 and NGC 4755, and will serve as standards against which our subsequent FLAMES observations in the Magellanic Clouds will be compared.
\end{abstract}

Key words. stars: early-type - stars: fundamental parameters - open clusters and associations: individual: NGC 3293 open clusters and associations: individual: NGC 4755 - open clusters and associations: individual: NGC 6611

\section{Introduction}

The Fibre Large Array Multi-Element Spectrograph (FLAMES) at the Very Large Telescope (VLT) provides multi-object spectroscopy over a corrected $25^{\prime}$ diameter field-of-view. As part of a European Southern Observatory (ESO) Large Programme, comprising over $100 \mathrm{~h}$ of VLT time,

\footnotetext{
* Based on observations at the European Southern Observatory in programmes 171.0237 and 073.0234 .

$\star \star$ Tables 9, 10, 11 and 12 are only available in electronic form at http://www. edpsciences.org
}

we have observed in excess of 50 O-type stars and 500 B-type stars, in a total of seven clusters distributed over the Galaxy and the Magellanic Clouds. This unprecedented survey of massive stars, and its homogeneous spectroscopic analysis using state-of-the-art model atmospheres, will be the subject of a series of papers. This first paper gives an overview of the scientific goals of the project, discusses the data reduction techniques employed for the survey, and presents the data for the three Galactic clusters.

A diverse range of topics depend critically on our understanding of the evolution of massive stars. Through their stellar 
winds and as supernova explosions they are the main source of kinetic energy into the interstellar medium of their host galaxies, and they are responsible for the continuous build-up of elements heavier than helium ("metals") from successive generations of star formation (e.g. Shapley et al. 2004). Indeed, one of the key challenges is to fully understand the role of metallicity on stellar evolution, to place better constraints on extremely low-metallicity stellar models (e.g. Kudritzki 2002). Theoretical simulations of star formation in the early Universe have suggested that masses larger than $100 M_{\odot}$ are strongly favoured (Bromm et al. 2002; Abel et al. 2002), and these "zero-metallicity" (or Population III) stars are considered to be prime candidates for the re-ionization of the Universe (e.g. Haehnelt et al. 2001; Wyithe \& Loeb 2003) that may have happened at redshifts greater than six (Becker et al. 2001).

Furthermore, with ongoing studies of the nature of supernova progenitors (e.g. Smartt et al. 2004), and following recent discoveries that some of the most spectacular supernovae appear to be related to $\gamma$-ray bursts (e.g. Galama et al. 1998; Bloom et al. 2002; Hjorth et al. 2003), understanding the evolution of massive stars will gain even greater importance in the near future. However, fundamental problems remain as to how the evolution of massive stars depends on the physics of massloss and rotation, and the role of metallicity. The FLAMES survey of massive stars aims at a comprehensive study of these processes. Some of the main scientific issues that we will address with the project are:

- Stellar rotation and chemical abundances: the inclusion of the effects of stellar rotation in evolutionary models leads to the important prediction of enhanced surface abundances of helium and nitrogen (e.g. Heger \& Langer 2000; Maeder \& Meynet 2000), with the enhancements predicted to be more significant at lower metallicity (Maeder \& Meynet 2001). Results from model atmosphere analyses of O- and B-type supergiants in the Magellanic Clouds (Crowther et al. 2002; Bouret et al. 2003; Hillier et al. 2003; Evans et al. 2004a; Trundle et al. 2004; Walborn et al. 2004; Trundle \& Lennon 2005) find evidence of significant nitrogen enrichment, that could in principle be attributed to mixing occurring in stars with initially high rotational velocities. However, none of the stars analysed to date have particularly large projected rotational velocities and it seems likely that either they have "spun down" more quickly than the models predict, or that rotationally-induced mixing is more effective than expected, even at relatively moderate velocities and at earlier evolutionary phases. The FLAMES survey will be used to determine stellar rotational velocities and to then provide a detailed study of their correlation with helium and $\mathrm{CNO}$ abundances.

- The dependence of stellar wind mass-loss rates on metallicity: accurate interpretation of the integrated spectra of starbursts (e.g., Vázquez et al. 2004) and high-redshift galaxies (e.g., Pettini et al. 2002; Rix et al. 2004) requires detailed knowledge of the dependence of stellar mass-loss rates, $\dot{M}$ with metallicity. Radiatively driven wind theory predicts that $\dot{M}$ should scale as $Z^{0.5-0.7}$ (e.g., Kudritzki et al. 1987; Vink et al. 2001). Observational evidence of this is still
Table 1. Galactic and Magellanic Cloud clusters observed with FLAMES. The earliest main-sequence object observed in each cluster is given as an indication of their relative ages.

\begin{tabular}{lll}
\hline \hline Cluster & Galaxy & Earliest main-sequence type \\
\hline NGC 3293 & Milky Way & B1 V (Feast 1958) \\
NGC 4755 & Milky Way & B0.5 V (Feast 1963) \\
NGC 6611 & Milky Way & O5 V (Hillenbrand et al. 1993) \\
NGC 330 & SMC & B0 V (Lennon et al. 1993) \\
NGC 346 & SMC & O4 V (Walborn et al. 2000) \\
NGC 2004 & LMC & early B \\
LH9/10 & LMC & O3 V (Parker et al. 1992) \\
\hline
\end{tabular}

rather qualitative (e.g. Evans et al. 2004c) and recent results in the Magellanic Clouds (e.g., Evans et al. 2004a; Trundle et al. 2004) have stressed the need for a large, well-sampled, homogenous study. The FLAMES sample will yield massloss rates (and chemical abundances) for a significant number of early-type stars in different environments, and will be well-suited to test the metallicity dependence; this is essential if we are to correctly include its effects in stellar evolution models and, ultimately, in population synthesis codes.

- Calibration of the wind-momentum luminosity relationship (WLR): the dependence of the modified wind momentum on stellar luminosity for OBA-type stars (e.g., Kudritzki et al. 1995; Puls et al. 1996; Kudritzki et al. 1999) potentially offers a purely spectroscopic method of distance determination in the Local Group and beyond. Recent analyses by Markova et al. (2004) and Repolust et al. (2004) have revisited this relationship for Galactic O-type stars; the FLAMES survey will enable a more precise calibration of this relationship in the Clouds.

The clusters observed with FLAMES are summarized in Table 1. The earliest (main sequence) spectral types observed in each cluster are included to give an indication of their relative ages. A typical FLAMES field yields 110-120 targets and at each metallicity we have targeted a young cluster and an older cluster (two in the case of the Milky Way).

In the current paper we discuss the methods used for target selection and detail the data reduction techniques employed for the survey. We also present spectral classifications, radial velocities and cross-identifications with previous catalogues for our targets in the three Galactic clusters: NGC 3293, NGC 4755 ( $a k a$ The Jewel Box), and NGC 6611 ( $a k a$ The Eagle Nebula; Messier 16). The data presented here also include observations of the brightest cluster members with the Fibre-Fed, Extended Range Optical Spectrograph (FEROS) at the 2.2-m Max Planck Gesellschaft (MPG)/ESO telescope. A thorough analysis of the rotational velocities of the Galactic sample will be given elsewhere (Lee et al. in preparation) and similar catalogues for the Magellanic Cloud targets will be presented in a further publication (Evans et al. in preparation). 
Table 2. Summary of the relevant EIS pre-FLAMES observations of Galactic fields used for target selection and astrometry.

\begin{tabular}{lccccll}
\hline \hline Cluster & EIS field & \multicolumn{2}{c}{ Field Centre (J2000) } & Date & \multicolumn{2}{c}{ Exposure time (s) } \\
& & $\alpha$ & $\delta$ & & $B$ & $V$ \\
\hline NGC 3293 & OC15 & 103528.0 & -582114 & $2000-02-26$ & $10+30+240+240$ & $10+30+240+240$ \\
NGC 4755 & OC22 & 125313.0 & -602806 & $2000-02-26$ & $30+240+240$ & $30+240+240$ \\
NGC 6611 & OC32 & 181851.9 & -134636 & $2000-07-29$ & $30+240+240$ & $30+240+240$ \\
\hline
\end{tabular}

\section{Target selection}

\subsection{WFI photometry}

Astrometry and photometry for our target fields were acquired from observations for the ESO Imaging Survey (EIS) that used the Wide Field Imager (WFI) at the 2.2-m MPG/ESO telescope (Momany et al. 2001). The relevant $B$ (B/99, ESO\#842) and $V(V / 89$, ESO\#843) science and calibration images were taken from the ESO archive and reduced using a modified version of the Isaac Newton Telescope-Wide Field Camera (INT-WFC) data reduction pipeline (Irwin \& Lewis 2001). Multiple exposures of the fields (aimed at shallow and deeper magnitudes) were combined to produce the final photometry; the observations are summarised in Table 2.

Photometric transformations for the WFI data to the Johnson-Cousins system were determined for each cluster from published photometry, using visually matched stars - in the absence of observations of standard fields this "bootstrap" approach was both necessary and sufficient for our requirements. The brightest targets in each cluster were saturated, even in the short WFI exposures, although the astrometry of these sources was more than adequate for positioning of the FLAMES fibres. The photometric transformations were determined using those targets that displayed a linear response i.e., $V>10.75^{\mathrm{m}}$ in NGC 3293 and $V>11.5^{\mathrm{m}}$ in NGC 4755 and NGC 6611.

In Figs. 1 and 2 we show fits to the colour terms in $V$ and $B$; published photometry was taken from Turner et al. (1980, NGC 3293), Sanner et al. (2001, NGC 4755), and Hillenbrand et al. (1993, NGC 6611). The transformation equations found (from 51 stars) for NGC 3293 were:

$V_{\mathrm{J}}=V_{\mathrm{WFI}}-0.09 \times(B-V)_{\mathrm{J}}-0.07$,

$B_{\mathrm{J}}=B_{\mathrm{WFI}}+0.25 \times(B-V)_{\mathrm{J}}-0.21$.

Similarly, the solutions (from 48 stars) for NGC 4755 were:

$V_{\mathrm{J}}=V_{\mathrm{WFI}}-0.06 \times(B-V)_{\mathrm{J}}-0.19$,

$B_{\mathrm{J}}=B_{\mathrm{WFI}}+0.29 \times(B-V)_{\mathrm{J}}-0.33$.

Lastly, the solutions (from 75 stars) for NGC 6611 were:

$V_{\mathrm{J}}=V_{\mathrm{WFI}}-0.04 \times(B-V)_{\mathrm{J}}-0.16$,

$B_{\mathrm{J}}=B_{\mathrm{WFI}}+0.32 \times(B-V)_{\mathrm{J}}-0.53$.

These colour terms are comparable to those found by Momany et al. (2001) for the pre-FLAMES WFI survey. In comparison with previously published values, after transformation we find mean (absolute) differences of $\sim 0.03^{\mathrm{m}}$, with $\sigma \sim 0.03^{\mathrm{m}}$ for both $V$ and $(B-V)$ in NGC 3293 and NGC 4755; in NGC 6611 we find differences of $0.04^{\mathrm{m}}$, with $\sigma=0.04^{\mathrm{m}}$ in both $V$ and $(B-V)$. Such accuracy is adequate for our purposes of target selection. We note that the fit for NGC 3293 in Fig. 1 is not biassed by the one star at $(B-V)>1$; the same colour term is found regardless of its inclusion.

Following transformation to the standard system, the observed stars in each cluster were assigned running numbers on the basis of the $V$-band magnitudes, with "3293-001" the brightest star observed in NGC 3293 and so on. This is not strictly the case for the stars in NGC 6611, following refinement of the calibrations (using an enlarged sample of published values) after the assignment of the running numbers.

In the process of determining the photometric transformations we noticed that two stars in NGC 4755, namely IV-11 and IV-17 in the numbering system of Arp \& van Sant (1958), are listed by Sanner et al. (2001) as having $V \sim 14^{\mathrm{m}}$. These are significantly discrepant from the (albeit photographic) values given by $\operatorname{Arp} \&$ van Sant ( $V=11.35$ and $9.76^{\mathrm{m}}$ respectively). Photographic (IV-11: $V=11.41^{\mathrm{m}}$ ) and photoelectric (IV-17: $V=9.96^{\mathrm{m}}$ ) results from Dachs \& Kaiser (1984) roughly tally with the Arp \& van Sant values, and the WFI observations confirm that they are much brighter than 14th mag. We suggest that these may be misidentifications by Sanner et al. (2001) rather than significant, intrinsic variability.

More curious is the case of Walker 442 in NGC 6611, with Thé et al. (1990) reporting $V=8.26$. The photographic observations by Walker $\left(1961, V=15.09^{\mathrm{m}}\right)$ and the value from the WFI data $\left(V=14.99^{\mathrm{m}}\right)$ are both much fainter for this star. Though the star is not explicitly cross-referenced, Hillenbrand et al. (1993) also give $V=15.00$ for a star at $\alpha=18^{\mathrm{h}} 19^{\mathrm{m}} 0.86^{\mathrm{s}}$, $\delta=-13^{\circ} 48^{\prime} 47.2^{\prime \prime}(\mathrm{J} 2000)$, almost certainly W442. It is hard to reconcile these observations with the The et al. (1990) value which, if real, would make W442 one of the visually brightest stars in the cluster. The sources of this discrepancy are not clear, but we note that the SIMBAD database reports the The et al. results.

\subsection{FLAMES-Giraffe spectroscopy}

The primary element of FLAMES is the Giraffe spectrograph. Our programme employed the mode of Giraffe in which it is fed by 132 so-called "Medusa" fibres. Input catalogues of possible targets were compiled for each cluster from the WFI photometry and astrometry; these were then used to allocate the Medusa fibres with the FLAMES Fibre Positioner Observation 


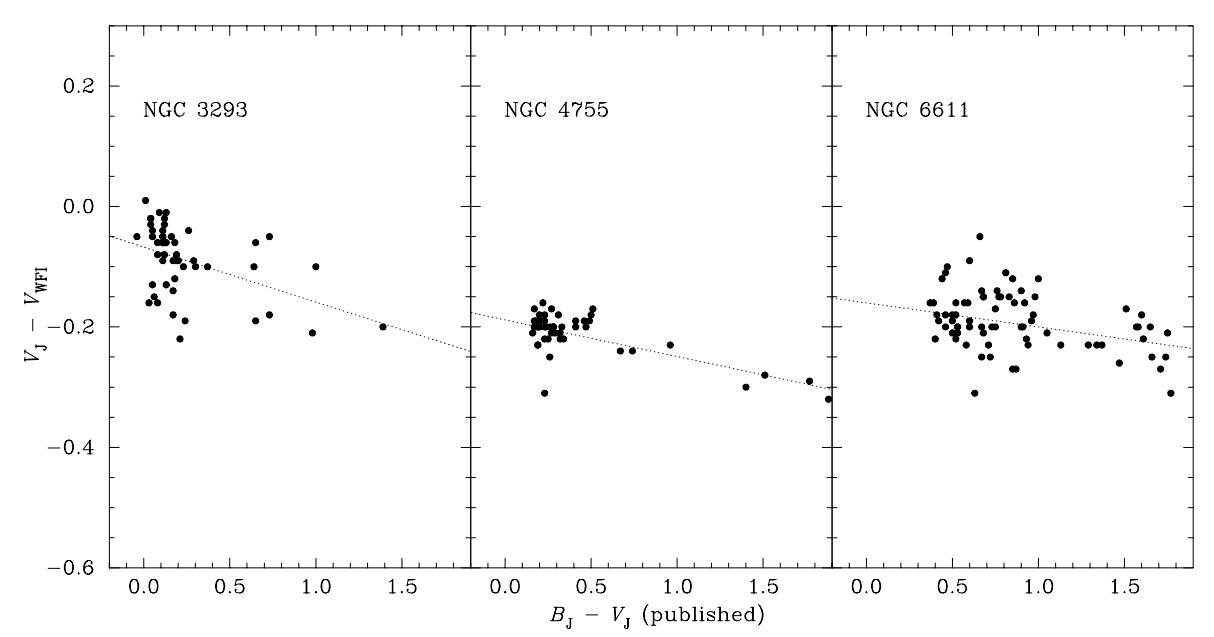

Fig. 1. Comparison of $V_{\mathrm{J}}-V_{\mathrm{WFI}}$ with published colours from Turner et al. (1980, NGC 3293), Sanner et al. (2001, NGC 4755) and Hillenbrand et al. (1993, NGC 6611). In addition to the zero-point offset, there is a relatively weak colour term between the WFI data and published results.

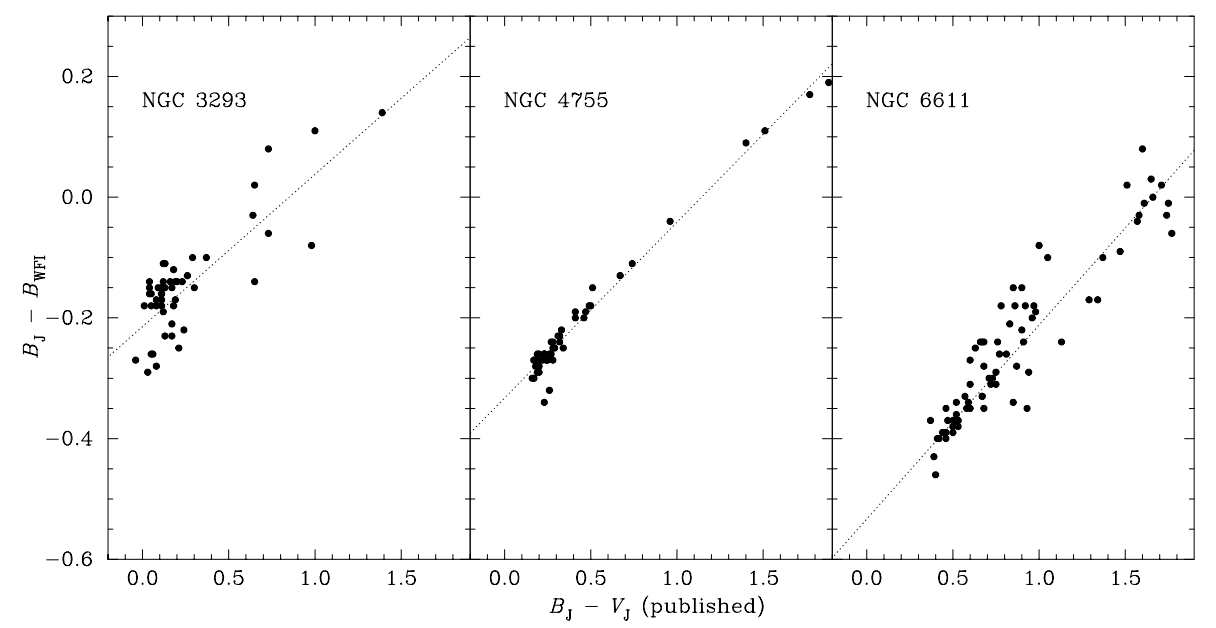

Fig. 2. Comparison of $B_{\mathrm{J}}-B_{\mathrm{WFI}}$ with published colours from Turner et al. (1980, NGC 3293), Sanner et al. (2001, NGC 4755) and Hillenbrand et al. (1993, NGC 6611). In addition to the zero-point offset, there is a colour term between the WFI data and published results.

Support Software (FPOSS). The philosophy behind compilation of Giraffe targets was to select bright blue stars, with cuts of $(B-V)_{0}<0.00^{\mathrm{m}}$ (corresponding to the threshold between B and A spectral types) and to a faint limit such that the main sequence was sampled down to $\sim$ B5 (corresponding to $\left.M_{V} \sim-1\right)$.

An important consideration for application of these selection criteria in the Galactic clusters is an estimate of the extinction and reddening. Estimates of $E(B-V)$ were incorporated into our selection routines, adopting $E(B-V)=0.30^{\mathrm{m}}$ for NGC 3293 (e.g., Turner et al. 1980) and $0.40^{\mathrm{m}}$ for NGC 4755 (e.g., de Waard et al. 1984). The treatment of reddening in NGC 6611 was somewhat more complicated; the extinction is large and strongly variable across the cluster and a value of $E(B-V)=1.20^{\mathrm{m}}$ was adopted, which is at the upper end of those found by Hillenbrand et al. (1993). This value ensured inclusion of the majority of the early-type objects (necessary for a reasonable density of targets across the FLAMES field-of-view), but contamination by (less-reddened) later-type stars can then become a problem. Indeed, as demonstrated by the spectral types present in our final sample (see Table 11), selection of solely early-type objects in such a cluster is extremely difficult without previous spectroscopic information.

Following compilation of the input catalogues, FPOSS was used to assign the Medusa fibres. In addition to selection effects such as avoiding fibre collisions and the physical size of the fibre buttons, the final spectral samples are influenced by some additional external constraints. Where published spectral types were available (and the object was of interest) a higher weight was assigned to the target when using FPOSS. Similarly, known Be-type stars were (in general) deliberately omitted from the input catalogues used with FPOSS; this is primarily relevant to configuration of our Magellanic Cloud fields but is mentioned here to note that this survey is not suitable for statistical studies of the incidence of Be-type stars.

The brightest stars in each cluster were deliberately omitted from the FLAMES observations as they were too bright for the dynamic range of the instrument; such stars would simply saturate during the exposure times needed for the fainter B-type main-sequence stars. These stars were observed separately using FEROS at the 2.2-m MPG/ESO telescope (see Sect. 2.3). 


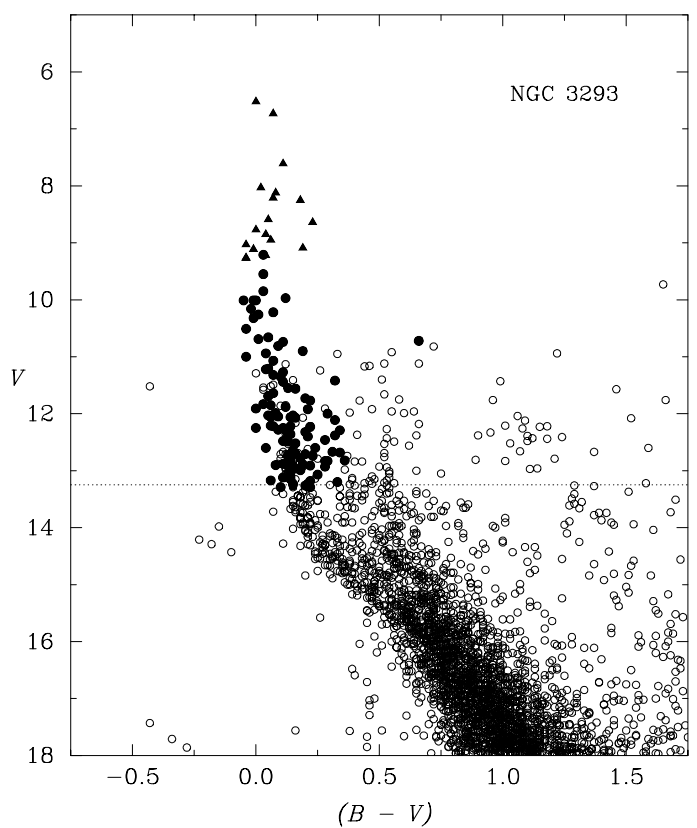

Fig. 3. Colour-magnitude diagram for all objects in the FLAMES field-of-view for NGC 3293. The marked targets were observed with FLAMES-Giraffe (solid circles) or with FEROS (solid triangles).

The colour-magnitude diagram for all the stars in the FLAMES field-of-view centered on NGC 3293 is shown in Fig. 3. Magnitudes and colours for stars fainter than $V=$ $10.75^{\mathrm{m}}$ are from our WFI photometry (corrected to the Johnson system); with photometry for the brighter stars from Turner et al. (1980) and Feinstein \& Marraco (1980). The faint limit of our targets $\left(V \sim 13.25^{\mathrm{m}}\right)$ is marked by the dotted line. The colour-magnitude diagrams for NGC 4755 and 6611 are shown in Figs. 4 and 5, with the photometry for the brightest stars from Sanner et al. (2001) and Hillenbrand et al. (1993) respectively.

\subsection{FEROS spectroscopy}

The brightest targets in NGC 3293 and NGC 4755 were observed using FEROS at the ESO/MPG 2.2-m telescope on 2004 July 4-6. We also sought observations of remaining targets of interest in the NGC 6611 field and, to increase the number of Galactic O-type stars in the survey, we included three stars classified as O-type by Hillenbrand et al. (1993) that were not in the FLAMES field-of-view (6611-001, 6611-005, and 6611-045; W412, HD 168504, and W584 respectively).

FEROS is a fixed configuration instrument (with $R=$ 48 000), giving a wide wavelength coverage of 3600-9200 $\AA$ in one exposure. The FEROS data were reduced using the reduction pipeline that runs under the MIDAS environment (Kaufer et al. 1999). In the majority of cases the signal-to-noise ratio of the FEROS data is in excess of 100 per resolution element. Targets observed with FEROS are marked in Figs. 3-5 as solid triangles.

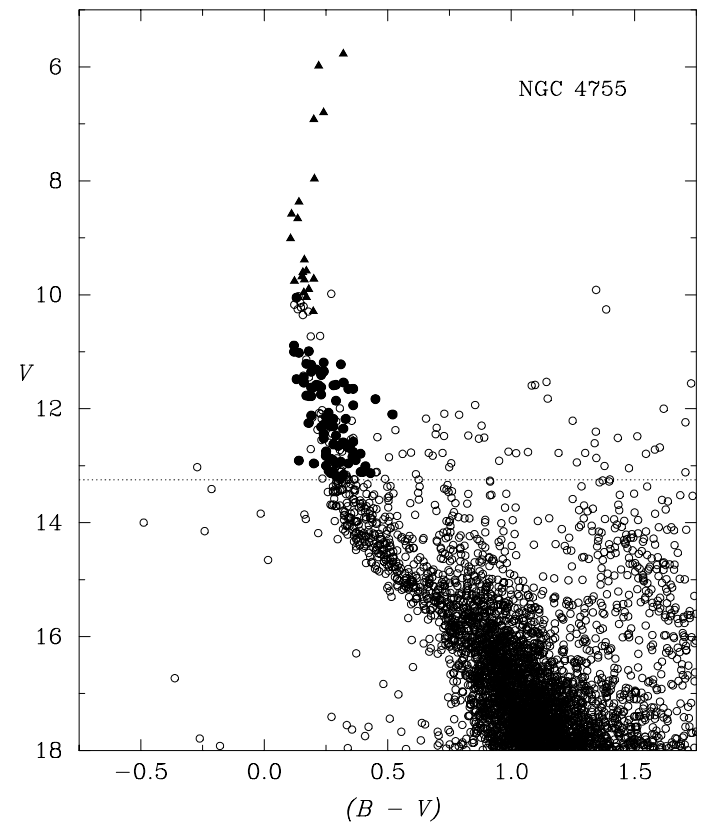

Fig. 4. Colour-magnitude diagram for all objects in the FLAMES field-of-view for NGC 4755. The marked targets were observed with FLAMES-Giraffe (solid circles) or with FEROS (solid triangles).

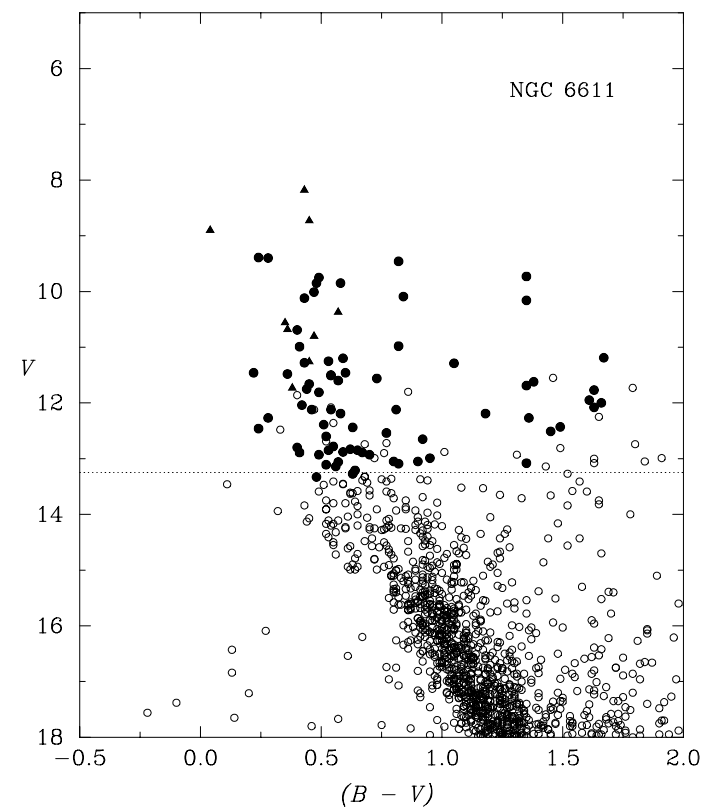

Fig. 5. Colour-magnitude diagram for all objects in the FLAMES field-of-view for NGC 6611. The marked targets were observed with FLAMES-Giraffe (solid circles) or with FEROS (solid triangles).

\section{FLAMES-Giraffe observations and data reduction}

Each of the three Milky Way clusters was observed with one FLAMES fibre configuration, centered on the cluster core. Six of the standard high-resolution Giraffe settings were used: HR02 (with a central wavelength of $\lambda 3958 \AA$ ),

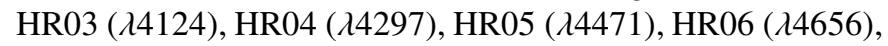
and HR14 ( 26515$)$. The spectral range for each setting is approximately $200 \AA$, i.e. there is sufficient overlap between 
Table 3. Summary of VLT-FLAMES observations of Galactic clusters. The exposure times are for each wavelength setting.

\begin{tabular}{lcclcl}
\hline \hline Cluster & \multicolumn{2}{c}{ Field centre (J2000.0) } & Date & Exp. time & Giraffe settings \\
& $\alpha$ & $\delta$ & & $(\mathrm{s})$ & \\
\hline NGC 3293 & 103548.7 & -581334 & $2003-04-14$ & 795 & HR02/03/04/05/06/14 \\
NGC 4755 & 125342.0 & -602215 & $2003-04-13$ & 795 & HR02/03/04/05/06/14 \\
NGC 6611 & 181850.0 & -134644 & $2003-07-15$ & $2 \times 375$ & HR02/03/04/05/06/14 \\
NGC 6611 & 181850.0 & -134644 & $2003-07-18$ & $2 \times 375$ & HR05/06/14 \\
\hline
\end{tabular}

settings to give a continuous spectrum of 3850-4755 $\AA$, with additional coverage of 6380-6620 ̊, primarily to observe the $\mathrm{H} \alpha$ Balmer line. The observations are summarized in Table 3, with details of the individual Galactic targets given in Tables 9-11.

The Galactic FLAMES data were reduced using IRAF $^{1}$ (v2.11). Standard routines were used for bias subtraction, fibre extraction and wavelength calibration. To remove the flat-field response from the science data, each extracted spectrum was divided through by the normalised flat-field spectrum (taken as part of the daytime calibrations) from the same fibre. Further manipulation of the data was undertaken using the STARLINK package DIPSO.

The Galactic targets are relatively bright and sky subtraction was not a critical issue, however such methods were fully explored for these data as it will be more important for the fields observed in the Magellanic Clouds. A master sky spectrum was created from merging the available sky spectra, rejecting those contaminated with a significantly larger than average Balmer line emission component or those contaminated by arc lines (see Sect. 3.1). In a cluster such as NGC 6611 there is considerable variation in the nebular emission across very small scales (let alone across the FLAMES field-of-view) and the subtraction of such features will not be $100 \%$ accurate; an inherent drawback of multi-fibre spectrscopy. The logic behind rejecting those sky spectra with significantly large nebular emission is driven by our desire to minimise over-subtraction in the line cores, although this proved unavoidable in some of the NGC 6611 targets. The extracted flat-field fibres were used to determine relative throughputs (compared to the median number of counts) for each fibre; the master sky spectrum was then appropriately scaled prior to subtraction from the science spectrum.

The exact resolving power of the grating varies with the different central wavelength settings. The mean $F W H M$ of the arc-lines in the central science fibre at each setting (averaged over the three observed fields) is summarized in Table 4. The typical signal-to-noise of the data ranges from 100-150 per resolution element.

For classification purposes the spectra for each region were rectified automatically (using pre-defined continuum regions) and then merged (using DIPSO routines developed by I. D. Howarth for echelle/fibre reductions) to give a

\footnotetext{
${ }^{1}$ IRAF is distributed by the National Optical Astronomy Observatories, which are operated by the Association of Universities for Research in Astronomy, Inc., under cooperative agreement with the National Science Foundation.
}

Table 4. Summary of the mean $F W H M$ of the arc lines and effective resolving power, $R$, at each Giraffe central wavelength setting, $\lambda_{\mathrm{c}}$.

\begin{tabular}{ccccc}
\hline \hline Setting & $\lambda_{\mathrm{c}}$ & \multicolumn{2}{c}{$F W H M$} & $R$ \\
& $(\AA)$ & $(\AA)$ & (pixels) & \\
\hline HR02 & 3958 & 0.19 & 3.7 & 20850 \\
HR03 & 4124 & 0.15 & 3.6 & 27500 \\
HR04 & 4297 & 0.19 & 3.6 & 22600 \\
HR05 & 4471 & 0.16 & 3.5 & 27950 \\
HR06 & 4656 & 0.20 & 3.6 & 23300 \\
HR14 & 6515 & 0.22 & 3.6 & 29600 \\
\hline
\end{tabular}

continuous spectrum of 3850-4755 $\AA$, with additional coverage of 6380-6620 А.

\subsection{Contamination by simultaneous arc-line fibres}

For programmes that demand high-accuracy radial velocity measurements there are five Medusa fibres assigned for simultaneous arc calibrations. These are distributed evenly across the CCD and (in the absence of information to the contrary at the time) were left enabled for the Galactic observations. Inspection of these arc spectra showed no significant wavelength shifts (for a given central wavelength) in comparison to the daytime calibrations. However, these checks did highlight the overspill of the simultaneous arc spectra into those from adjacent fibres on the CCD. Several of our science spectra contain arc-line contamination from this overspill, although the contamination is generally weak and in the majority of cases limited to the red (HR14, 26515) wavelength setting.

\subsection{Comparison with pipeline reductions}

During the course of this survey, the Giraffe Base-Line Data Reduction Software (girBLDRS) underwent significant development at Observatoire de Genève (see Blecha et al. 2003, for full details); this pipeline has been used for reduction of the Magellanic Cloud observations (Evans et al. in preparation).

Prior to reduction of the Magellanic Cloud data, we re-reduced the observations in NGC 3293 using girBLDRS (v.1.10) for comparison with the IRAF reductions. In brief, we used the calibration data observed at the time of our science frames to update those released with girBLDRS, and then used the "extract" pipe. The pipeline default is an optimal extraction of each fibre, however this option relies on correction of the localisation of the fibres on 
Table 5. Overview of the wavelength coverage and resolution obtained from the different instrumentation employed in the current study.

\begin{tabular}{lcc}
\hline \hline Spectrograph & Wavelength coverage $(\AA)$ & $R$ \\
\hline FLAMES-Giraffe & $3850-4755,6380-6620$ & $20850-29600$ \\
FEROS & $3600-9200$ & 48000 \\
WHT-ISIS & $3800-5100,6200-6800$ & 7000 \\
\hline
\end{tabular}

the CCD. The master localisation is obtained using the flatfield frames, but the correction employs the simultaneous arc spectra. In anticipation of the fact that the simultaneous arcs were disabled for the majority of our FLAMES observations in the Magellanic Clouds (because of the overspill discussed in Sect. 3.1), the simpler summed extraction method was used for our comparisons. After extraction with girBLDRS, a combined sky spectrum was subtracted from each target using DIPSO, employing the same methods as those used in the IRAF reductions. These re-reduced data were rectified using the same scripts as before and the final spectra are in excellent agreement.

\subsection{Comparison with longslit spectroscopy}

We have conventional longslit spectroscopy of the majority of our O-type targets in NGC 6611 (see Table 11). These were observed as part of separate programmes at the $4.2-\mathrm{m}$ William Hershel Telescope (WHT) on 2003 June 15-17 and October 16-17, using the Intermediate-dispersion Spectroscopic and Imaging System (ISIS) with 1200B and $1200 \mathrm{R}$ gratings. Observations were made at three central wavelength settings in both arms of ISIS, giving continuous spectral coverage of 3800-5100 $\AA$, with additional coverage of $6200-6800 \AA$, at $R \simeq 7000$. The basic properties of the ISIS observations are summarized in Table 5, in which they are compared with those from FLAMES-Giraffe and FEROS.

In general we find excellent agreement between the FLAMES-Giraffe and ISIS spectra (aside from detection of binarity, see Sect. 6). Indeed, minor differences in the final spectra arise in the far-blue region, not because of problems with the FLAMES spectra but from residual features from the ISIS dichroic used to simultaneously observe in both blue and red wavelength regions. Of particular note is that the equivalent widths of important diagnostic lines (such as He I $\lambda 4471$, $\mathrm{He}$ II $\lambda 4686$ and $\mathrm{H} \gamma$ ) agree, adding confidence to the multi-fibre FLAMES data.

In Fig. 6 we compare the $\mathrm{H} \alpha$ Balmer line profiles for the six stars in NGC 6611 observed with both FLAMES-Giraffe and WHT-ISIS; the FLAMES spectra have been degraded to the effective resolution of the WHT-ISIS data to ensure a more meaningful comparison. As one would expect from multifibre observations, the nebular subtraction is not perfect in the FLAMES spectra. However, the nebular component is easily resolved and the wings of the profiles are in good agreement with the longslit spectroscopy, and will still offer strong constraints on the physical parameters. The differences in Fig. 6 for 6611-011 and 6611-014 are accounted for by binarity, further discussed in Sect. 6.
Table 6. Total numbers of stars in each cluster by spectral type (incorporating both the FLAMES and FEROS observations).

\begin{tabular}{lccccccc}
\hline \hline Field & \multicolumn{9}{c}{ Spectral type } & & Total \\
& $\mathrm{O}$ & $B<5$ & $B \geq 5$ & A & F & GK & \\
\hline NGC 3293 & - & 48 & 51 & 24 & 3 & - & 126 \\
NGC 4755 & - & 54 & 44 & 9 & 1 & - & 108 \\
NGC 6611 & 13 & 28 & 12 & 10 & 10 & 12 & 85 \\
\hline
\end{tabular}

\section{Spectral classification}

The FLAMES-Giraffe spectra of our Galactic targets were classified in the MK system (Morgan et al. 1943) by visual inspection. The Giraffe spectra have a much greater resolution than those traditionally used in spectral classification, so the data were degraded to an effective resolution of $1.5 \AA$ and then classified according to published morphological precepts. Work is underway by other groups (e.g. Bagnulo et al. 2003) to compile high-resolution observations of spectral standards and our survey will significantly add to such efforts. Furthermore, some of our stars have robust spectral types in the literature and so also serve as internal standards for the current dataset. The classification criteria applied to each spectrum are summarized below, points of interest and spectral peculiarities are also discussed. Following initial classification by CJE, the data were reinspected to ensure internal consistency and, if necessary, minor revisions of spectral type were made. A subset of these data were independently classified by DJL to provide an external check on the spectral types; agreement to within one spectral subtype was found in all cases. The distribution of observed stars by spectral type is summarised in Table 6 .

\subsection{O-type spectra}

The O-type spectra were classified using the digital atlas of Walborn \& Fitzpatrick (1990) as the principal reference; the primary classification criterion is the ratio of He I to He II lines. A total of 13 O-type stars were observed, all of which are in NGC 6611. The standard suffixes are used to indicate the intensity of N III emission and the behaviour of He II $\lambda 4686$ (see Walborn \& Fitzpatrick for further details). FLAMES-Giraffe spectra of three O-type stars in NGC 6611 are shown in Fig. 7; note that 6611-080 is one of the faintest targets in the Galactic sample and yet the data are of sufficient quality to permit detailed analysis in a future study.

\subsection{B-type spectra}

The observed B-type spectra were classified from comparisons with the standards in the Walborn \& Fitzpatrick (1990) atlas, which extends as late as B3 for dwarfs. These standards were complemented by unpublished observations of B-type dwarfs using the Intermediate Dispersion Spectrograph (IDS), at the 2.5-m Isaac Newton Telescope (INT) in 1999 December, and with FEROS in 2004 July. These additional standards are summarized in Table 7. 


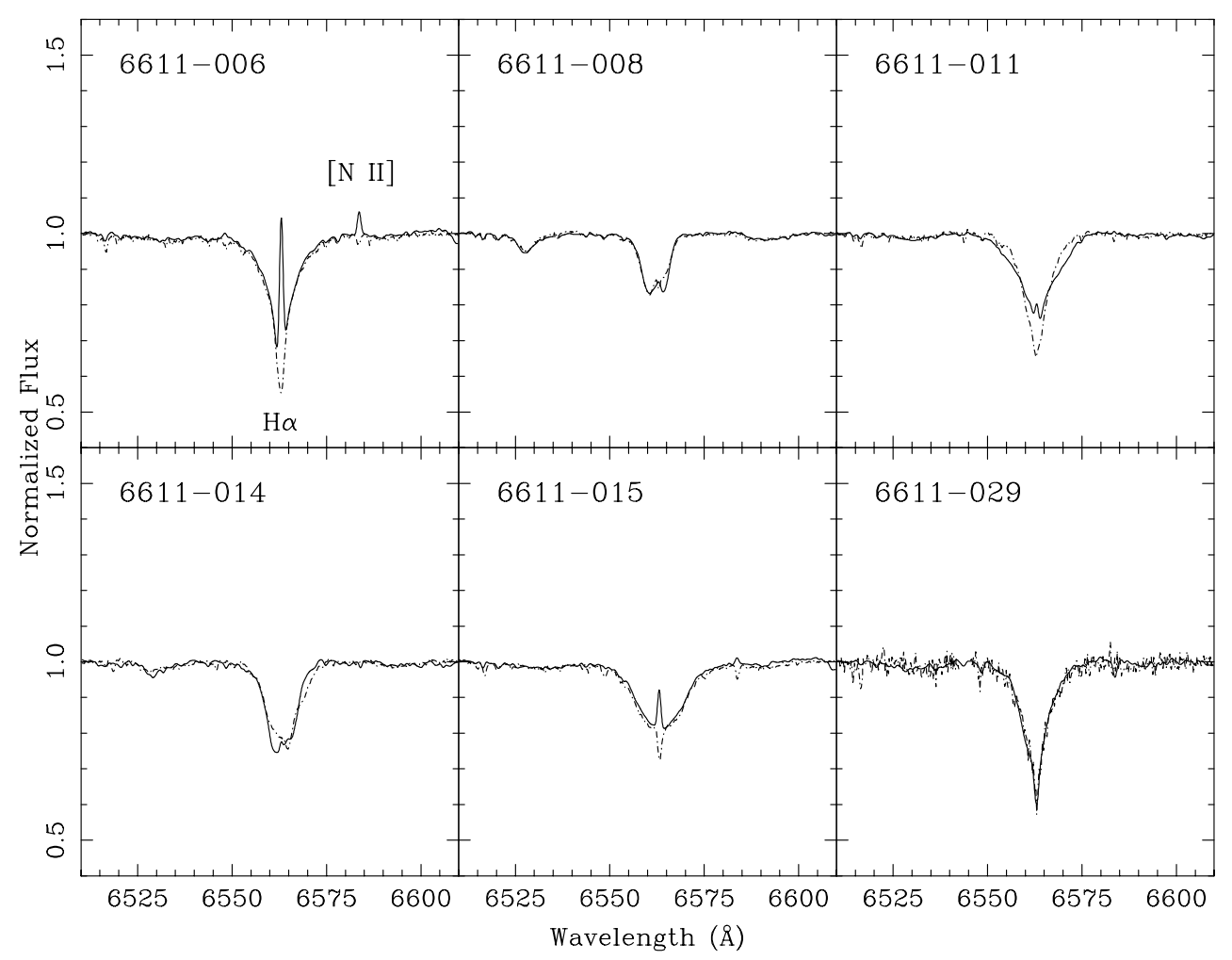

Fig. 6. Comparison of sky-subtracted FLAMES-Giraffe (solid line) and WHT-ISIS (dot-dashed line) H $\alpha$ profiles, in which the FLAMES data have been smoothed to the effective resolution of the ISIS spectra. The nebular subtraction in the FLAMES spectra is not perfect but its contribution is resolved. Note the lower signal-to-noise ratio in the ISIS spectrum of 6611-029, and the differences in 6611-011 and 6611-014, explained by binarity (see Sect. 6).

Some of the Giraffe spectra are assigned the non-unique spectral type of B6-7 (see Tables 9 and 10). Examples of B6 and B7-type supergiants were presented by Lennon et al. (1992), with subtle variations seen in the metallic spectra as one progresses from B5 through to B8. The FLAMES spectra however are largely of less-luminous objects and such subtleties are not seen because of the intrinsically weaker metallic lines in non-supergiants. Additionally, rotational velocities are typically larger in non-supergiant B-type stars, which further confuses the issue. As a result, here we classify those spectra that are clearly between the observed B5 and B8 standards as "B6-7".

The width of the lines in the B-type spectra offer evidence of a wide range of rotational velocities, some are particularly sharp-lined, whilst others are significantly broadened. For now we limit ourselves to simply adding the conventional " $n$ " suffix to those B-type spectra which display the most significant broadening, employed here when the He I $\lambda 4471$ and Mg II $\lambda 4481$ lines are strongly blended. A thorough analysis of the distribution of rotational velocities will be presented elsewhere (Lee et al. in preparation).

\subsubsection{Luminosity classification}

Luminosity types were assigned to the B-type spectra using measurements of the $\mathrm{H} \gamma$ equivalent widths and the calibrations of Balona \& Crampton (1974). In some cases the measured
Table 7. Summary of B-type classification standards in addition to those from Walborn \& Fitzpatrick (1990).

\begin{tabular}{llll}
\hline \hline Star & Sp. type & Telecope & Reference \\
\hline HD 198781 & B0.5 V & INT & Morgan et al. (MCW 950; 1955) \\
HD 24131 & B1 V & INT & Morgan et al. (MCW 251; 1953) \\
HD 215191 & B1.5 V & INT & Lesh (1968) \\
HD 212978 & B2 V & INT & Blaauw \& Morgan (1953) \\
HD 182180 & B2 Vn & ESO-2.2 & Hiltner et al. (1969) \\
HD 23625 & B2.5 V & INT & Lesh (1968) \\
HD 20365 & B3 V & INT & Roman \& Morgan (1950) \\
HD 25558 & B3 V & INT & Blaauw (1956) \\
HD 19268 & B5 V & INT & Lesh (1968) \\
HD 34233 & B5 V & INT & Lesh (1968) \\
HD 58715 & B8 V & INT & Morgan et al. (1943) \\
HD 224112 & B8 V & ESO-2.2 & Hiltner et al. (1969) \\
HD 107696 & B9 V & ESO-2.2 & Hiltner et al. (1969) \\
\hline
\end{tabular}

widths are mid-way between the published values for class III and V objects, resulting in an adopted class of "III-V".

Balona \& Crampton (1974) largely confirmed the earlier results of Petrie (1965) for B-type spectra. Their (photographic) work remains the most useful and reliable source of such calibrations, although some less wide-ranging studies (primarly concerned with absolute magnitudes of supergiants, of use in consideration of extragalactic distances) have made use of 


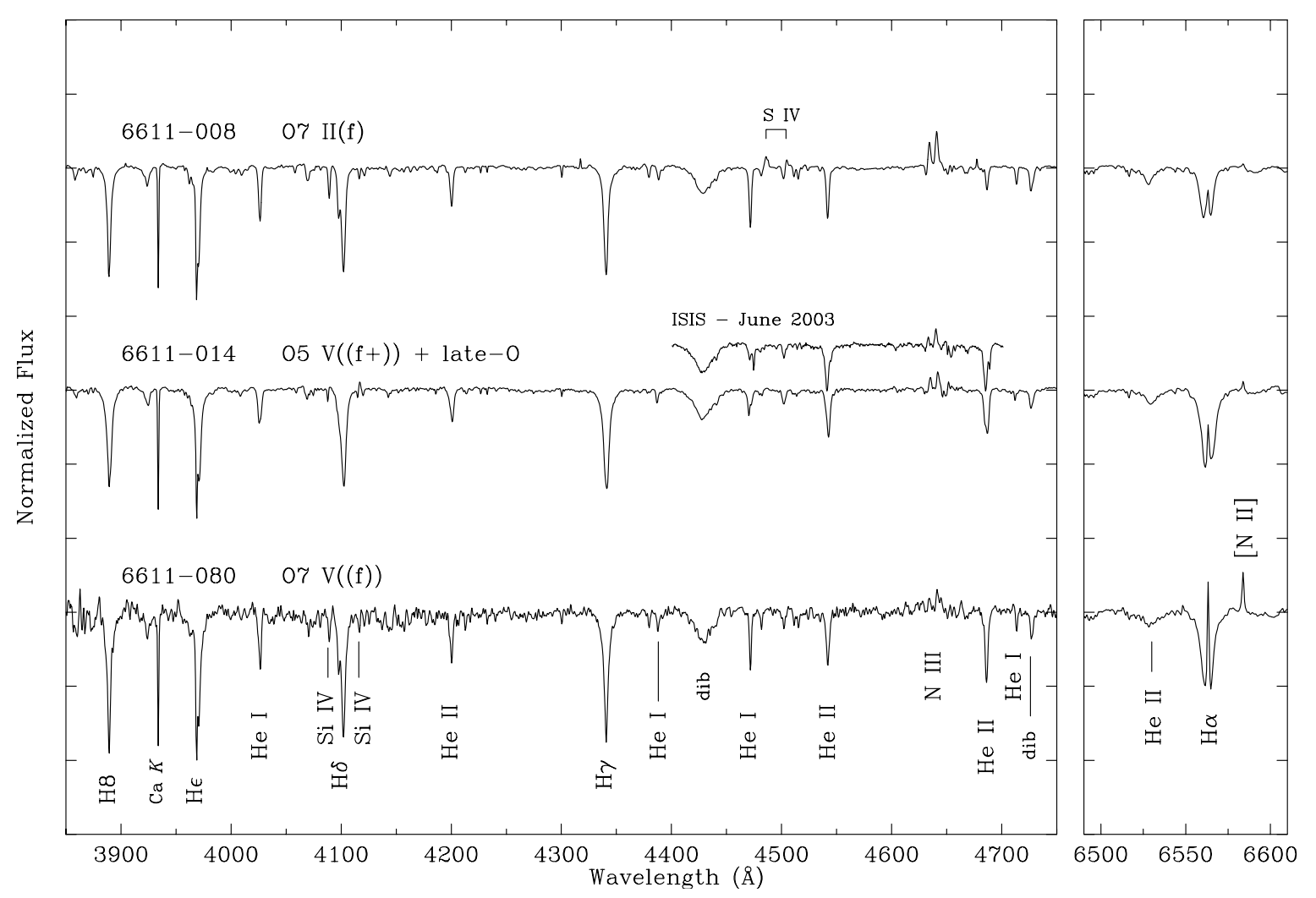

Fig. 7. Illustrative FLAMES O-type spectra in NGC 6611. For display purposes the spectra have been smoothed and rebinned to a resolution of $1.5 \AA$ FWHM and are offset by 0.75 continuum units. A section of the longslit ISIS spectrum of 6611-014 is also shown, illustrating its binarity (see Sect. 6). The sky spectrum has been subtracted from the blue-region data but, to highlight the nebular contribution, it has not been removed in the red. The spectral lines identified in 6611-080 are, from left to right by species, H I $\lambda \lambda 3889$ (H8), 3970 (H $\epsilon$ ), 4102 (H $\delta$ ), $4340(\mathrm{H} \gamma), 6563(\mathrm{H} \alpha)$; He I $\lambda \lambda 4026,4388,4471,4713$; He II $\lambda \lambda 4200,4541,4686,6527$; Si IV $\lambda \lambda 4089,4116$; N III $\lambda \lambda 4634-40-42 ;$ and the interstellar Ca $K \lambda 3933$ line. Also marked are the diffuse interstellar bands at $\lambda 4430,4726$ and nebular N II forbidden emission at $\lambda 6583$. The S IV $\lambda \lambda 4486,4504$ emission lines are marked in 6611-008.

more modern detectors (e.g. Hill et al. 1986). We envisage that with completion of our survey of Galactic and Magellanic Cloud targets we will be in a position to revisit these relations with an unprecedented (and fully homogenous) dataset. Indeed, two of the clusters observed by Balona \& Crampton (1974) were NGC 3293 and 4755; pending a new study we do not explore these issues further in the current work.

\subsection{A-type spectra}

Digital observations of A-type spectra were presented by Evans $\&$ Howarth (2003). Their principal classification criterion uses the intensity of the $\mathrm{Ca} \mathrm{K}$ line compared to the $\mathrm{H} \epsilon / \mathrm{Ca} \mathrm{H}$ blend. Although their work was principally motivated by studies of A-type supergiants, the adopted scheme was found to be largely insensitive to luminosity effects (e.g. their Fig. 4). However, reliance on the calcium line ratio is not without problems (see discussion by Evans \& Howarth), and with the high quality of the FLAMES data we are also able to give consideration to the metallic features in the spectra, which increase as one progresses to later-types. Thus, the observed A-type stars were classified using the criteria of Evans \& Howarth (2003), with secondary consideration to the intensity of the metal-lines.
Luminosity types were allocated using the $\mathrm{H} \gamma$ equivalent-width criteria from Evans et al. (2004b)

\subsection{Later-type spectra}

As a result of the significant and variable reddening in the direction of NGC 6611, our target selection methods yielded numerous (22 of 73) targets with spectral types of F0 or later. Without prior spectroscopy to make informed decisions in target selection, this is not a surprising result. It is not our intention to analyse these stars in the future, but they are included here for completeness. The classification bins applied to these later-type spectra are largely those described by Evans \& Howarth (2003), with additional reference to the useful online digital spectral atlas of R. O. Gray ${ }^{2}$, for those in the region G5-K2.

\subsection{Ho morphology}

Useful as a diagnostic of stellar winds and Be-type stars, when of particular note the morphology of the $\mathrm{H} \alpha$ profiles of our

\footnotetext{
${ }^{2}$ http://nedwww.ipac.caltech.edu/level5/Gray/ Gray_contents.html
} 
targets is summarized in the final column of Tables 9-11. The majority of the sample display $\mathrm{H} \alpha$ in absorption, with many of these also showing superimposed (narrow) nebular emission. Such stars would be best described as "abs+em" but are not explicitly noted in the tables - exceptions to this are 3293-001 and 4755-003 in which the emission is broader and more likely stellar in origin. The notation employed to describe the other $\mathrm{H} \alpha$ profiles is: "broad em" for broad emission; "twin" for twinpeaked emission (usually synonomous with disk-like features); "abs+twin" for those spectra in which twin-peaked emission is seen superimposed on an absorption profile; and "P Cyg" for stars showing a P Cygni emission profile.

\subsubsection{Emission line spectra}

A small number of emission line objects were observed in our Galactic fields, the majority of which can be categorized as normal Be-type spectra. Three spectra (namely 3293-022, 3293-045, and 4755-014) have strong twin-peaked $\mathrm{H} \alpha$ emission profiles; five (3293-027, 3293-040, 4755-038, 4755-050 and 4755-057) have twin-peaked emission visible in the wings of the $\mathrm{H} \alpha$ lines, superimposed on absorption profiles; and four (3293-011, 4755-018, 6611-010 and 6611-028) display broad, single-peaked emission features. These have all been classified as Be or Ae stars. Broad $\mathrm{H} \alpha$ emission is also seen in 6611-022, which is discussed further in Sect. 4.6.

Further caution regarding the problems of nebular subtraction is prompted by the significantly large number of Be/Ae objects in NGC 6611 reported by Hillenbrand et al. (1993). From observations with a slitless, grism spectrograph, Herbig \& Dahm (2001) attribute most of the emission features as due to nebular contamination, rather than from intrinsic stellar phenomena. For instance, the $\mathrm{H} \alpha$ emission in four targets reported as Be-type by Hillenbrand et al. is best described here as "absorption with narrow (presumably nebular) emission" (6611-020, 064, 066 and 068). Each of these is reported by Herbig \& Dahm as having $\mathrm{H} \alpha$ absorption profiles (albeit their data are at relatively low resolution); these results reinforce our cautious approach to employing the Be-notation

\subsection{Peculiar spectra}

A number of peculiarities were noted in the process of classification, which are now discussed in turn:

3293-034: the hydrogen lines and metallic spectrum are consistent with a spectral type of B2, but the He I lines are significantly enhanced (see Fig. 8) and it is therefore classified as B2 IIIh (cf. Walborn 1983).

3293-058: significantly enhanced Si II $\lambda 4128-32$ is seen in this spectrum, which is otherwise that of a normal A0-type (see Fig. 8). Such behaviour in the silicon lines is a well documented feature of Ap-type spectra (e.g. Morgan 1933) and the spectrum is classified as A0 IIp ( $\mathrm{Si}$ ).

3293-072: this star has a rich metallic spectrum and from the relative ratios of $\mathrm{He} \mathrm{I} / \mathrm{Mg}$ II and the intensity of the Si II lines is classified here as B8 II. However, some of the He I lines appear peculiar in that they appear more broadened and asymmetric than the relatively sharp metal lines (see Fig. 8). Inspection of the raw data (i.e., unsmoothed) in velocity space revealed no consistent component for the He I lines; although the plausible explanation for their peculiar appearance remains a hotter, though less luminous companion. In the absence of further information at the current time, we classify the spectrum as B8 IIp.

4755-092 and 4755-104: these two spectra also display enhanced Si II and are classified as A0 IIp (Si). In comparison to 3293-058, other metallic species also appear slightly more enhanced in these stars, e.g. lines from Fe II. The spectrum of 4755-092 is shown in Fig. 8.

6611-006: this star has been classified by a number of authors as O9.5 V (Hiltner \& Morgan 1969; Hillenbrand et al. 1993; Bosch et al. 1999) but from the FLAMES spectrum it is clear that the strength of the Si III triplet at $\lambda 4552-68-75$ is inconsistent with such a type. This star was subsequently observed with FEROS, with no obvious evidence of binarity found. Taking into account the effects of metallicity, the spectrum is similar to that of $\mathrm{AzV} 170$ in the SMC (Walborn et al. 2000) and we classify it here as O9.7 IIIp. There is no formal definition of the O9.7 type below class II (Walborn 1972) and a marginally later type of B0 IV (cf. the standards from Walborn \& Fitzpatrick 1990) might be more fitting; there is also a rich spectrum of carbon and oxygen lines. Pending further examination of the spectral standards in this domain, for now we employ the "p" suffix to denote peculiarity. To place this particular object in its broader context, it is the principal star seen in the now widely circulated Hubble Space Telescope (HST) Wide Field and Planetary Camera 2 (WFPC2) image of the "pillars" in the Eagle Nebula (Hester \& Scowen 1995, Arizona State University/NASA).

6611-022: the spectrum of 6611-022 (Walker \#235) is rich in Fe II emission features and displays significant, broad $\mathrm{H} \alpha$ emission. It is classified here (as by other authors e.g., Herbig \& Dahm 2001) as Herbig Be, following the identification of such stars by Herbig (1960). At the high resolution of our data we resolve two distinct peaks in each emission feature, strongly indicative of some form of rotating disk or shell around the star.

\subsection{Interstellar absorption lines}

Strong interstellar Ca II H and K lines at $\lambda 3933$ and $\lambda 3968$ are observed towards all three clusters, although blended with stellar features for the cooler targets. For the two older clusters (NGC 3293 and NGC 4755) the absorption is not resolved into separate components and probably arises mainly in the warm diffuse interstellar medium; no other interstellar lines are apparent although it should be noted that the spectral region containing the Na I doublet was not observed.

The interstellar spectra for the NGC 6611 targets are far richer, likely reflecting the substantial circumstellar and interstellar material associated with this young cluster. For example, Thé et al. (1990), Hillenbrand et al. (1993) and Yadav \& Sagar (2001) have all discussed both the variable 


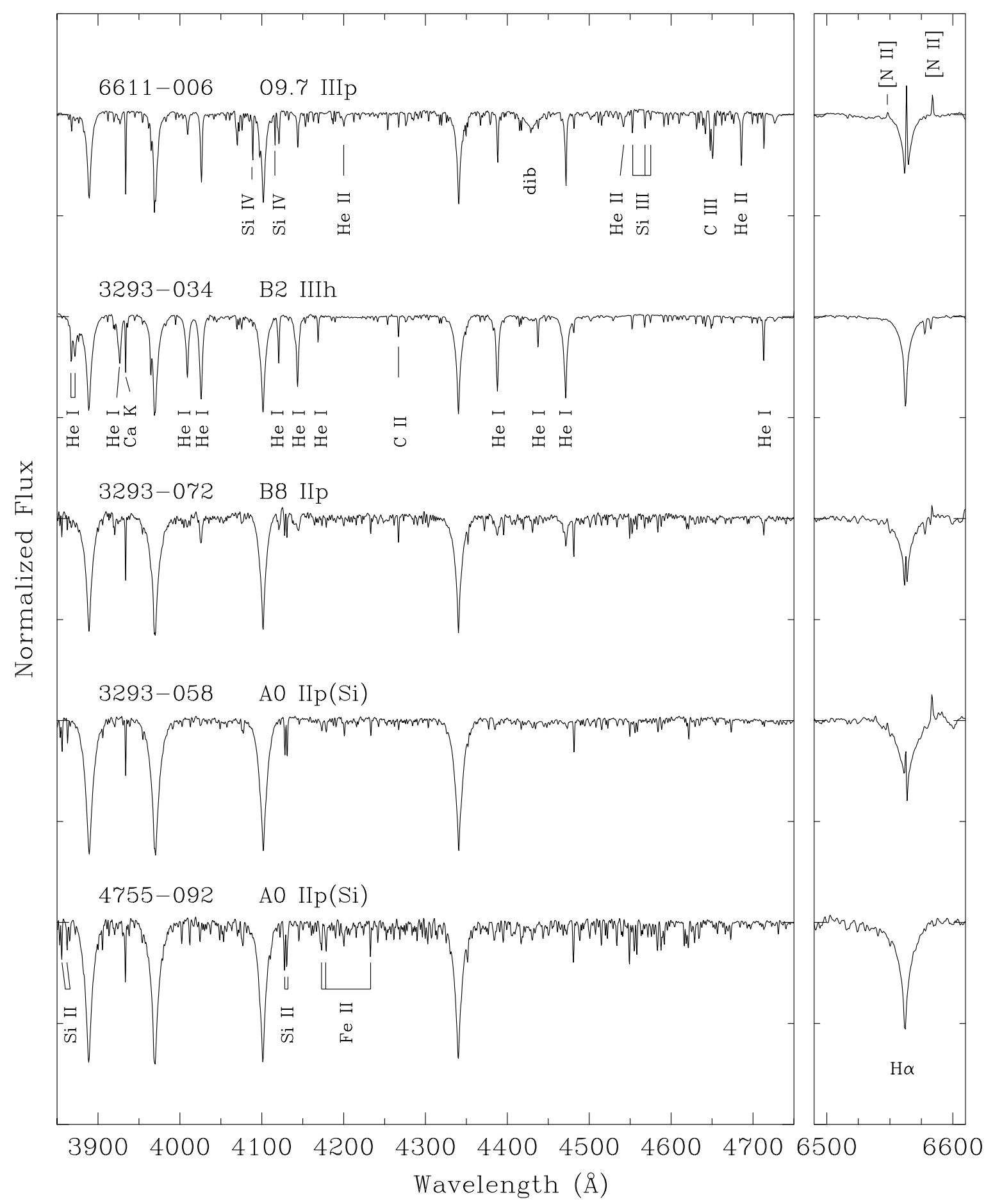

Fig. 8. FLAMES-Giraffe spectra of particular interest. The spectral lines identified in 6611-006 are, from left to right by species, He II $\lambda \lambda 4200$, 4541, 4686; Si III $\lambda \lambda 4553-68-75$; Si IV $\lambda \lambda 4089,4116$; the C III + O II blend at $\lambda 4650$; the N II forbidden emission lines at $\lambda \lambda 6548,6583$; and the diffuse interstellar band at $\lambda 4430$. The lines identified in 3293-034 are, He I $\lambda \lambda 3867+3872,3926,4009,4026,4121,4144,4169,4388$, 4438, 4471, 4713; C II $\lambda 4267$; and the interstellar Ca K line at $\lambda 3933$. Lastly, the metallic lines identified in 4755-092 are Si II $\lambda \lambda 3856,3862$, 4128-30; and Fe II $\lambda \lambda 4173-78,4233$. For display purposes the spectra have been smoothed and rebinned to a resolution of $1.5 \AA F W H M$ and are offset by 1 continuum unit.

extinction across the cluster and the non-standard reddening law. Furthermore, Welsh (1984) found evidence for high velocity interstellar components in IUE spectra. He interpreted these as arising from a shock-front (that is driven by stellar winds) interacting with the ambient neutral interstellar gas.
In the NGC 6611 FLAMES-Giraffe spectra, the Ca II lines show significant spatial variations - with a single strong component (which could be unresolved multiple components), complemented by up to four additional high velocity components. The strong component probably again reflects the warm 
diffuse interstellar medium, whilst the other features are likely to be spatially associated with the cluster.

Many sightlines show molecular absorption lines (with significant variations in the line strengths) from $\mathrm{CH}$ (14300), $\mathrm{CH}^{+}$( 24232$)$, and $\mathrm{CN}$ ( $\left.\lambda 3875\right)$, implying the presence of a cold molecular cloud. In some targets the Ca I $\lambda 4227$ line is also observed, further evidence of a dense, optically-thick cloud. Finally, He I absorption at $\lambda 3889$ (from the $2 s^{3} \mathrm{~S} \rightarrow 3 \mathrm{p}^{3} \mathrm{P}$ transition) is observed towards some objects, but with large spatial variations. This was first observed by Wilson (1937) and arises in an ionized plasma in which the helium ions are recombining to a metastable triplet state (e.g. Oudmaijer et al. 1997). The source of the ionization in this instance is presumably the strong X-ray continuum emitted by the O-type stars in the cluster.

\subsection{Comparisons with previous spectroscopy}

In general there is good agreement between our classifications and those extant in the literature, with many of the fainter stars now receiving more refined classifications, as one would expect from the new high-quality data. In Table 12 we give previous spectral types for our target stars. Published classifications are included from: Bidelman (1954); Bosch et al. (1999); Conti \& Leep (1974); Feast (1958, 1963); Hernández (1960); Hillenbrand et al. (1993); Hiltner et al. (1969); Hiltner \& Morgan (1969); Hoffleit (1956); Morgan et al. (1955); Schild (1970); Turner et al. (1980); Walker (1961); Walborn (1972, 1973, 1976, 1982). We omit the spectral types in NGC 6611 from de Winter et al. (1997) as they are largely based on photometric methods, and are thus "low weight" classifications.

Many of the targets in NGC 3293 (92 of 126 stars) and NGC 4755 (86 of 108 stars) do not have previous classifications from the sources compiled in Table 8. Even in NGC 6611, in which the spectral content has been well explored in the past (most significantly by Hillenbrand et al. 1993), we provide classifications for 31 stars without previous types in the literature. Our main science drivers do not require spectroscopic completeness but, to be thorough, we note that due to observational constraints (primarily the fact that only one field configuration was observed) a small number of B-type stars with published spectral types from Hillenbrand et al. (1993) are not included in the current survey.

\section{Stellar radial velocities}

Radial velocities $\left(v_{\mathrm{r}}\right)$ of our targets were measured from the Doppler shift of the $\mathrm{H} \gamma, \mathrm{H} \delta, \mathrm{H} \epsilon$, and $\mathrm{H} 8(\mathrm{H} \zeta)$ Balmer lines. This relatively simple approach (compared to using multiple metallic lines) was adopted to ensure internal consistency over the wide range of observed spectral types. The line centres were measured by Gaussian fits to the inner region of the profile, or by manual estimates of the position of minimum intensity. Only the inner part of the Balmer lines ( $\pm 3 \AA$ of the centre) were used for the Gaussian fits because of the effect of linear Stark broadening on the wings; the whole profile is poorly fit by a Gaussian function but, in general, the inner part is successfully matched. This approach worked well for symmetric
Table 8. The mean radial velocity of the clusters $\left\langle v_{\mathrm{r}}\right\rangle$, with their dispersions $\sigma_{v}$, and the mean error in the individual measurements $\left\langle\delta v_{\mathrm{r}}\right\rangle$. The number of stars, $n$, used for each cluster (after removal of outliers) is also given.

\begin{tabular}{lccc}
\hline \hline Cluster & $\begin{array}{c}\left\langle v_{\mathrm{r}}\right\rangle \pm \sigma_{v} \\
{\left[\mathrm{~km} \mathrm{~s}^{-1}\right]}\end{array}$ & $\begin{array}{c}\left\langle\delta v_{\mathrm{r}}\right\rangle \\
{\left[\mathrm{km} \mathrm{s}^{-1}\right]}\end{array}$ & $n$ \\
\hline NGC 6611 & $+10 \pm 8$ & 5 & 33 \\
NGC 3293 & $-12 \pm 5$ & 4 & 70 \\
NGC 4755 & $-20 \pm 5$ & 3 & 56 \\
NGC 4755 (bright) & $-14 \pm 8$ & 3 & 16 \\
NGC 4755 (faint) & $-21 \pm 5$ & 3 & 45 \\
\hline
\end{tabular}

profiles and was also particularly effective when weak nebular emission features were present in the line centres, because the fits are relatively unaffected. In cases where the lines were very sharp or asymmetric this approach was not successful and the line centres were determined from manual measurements. In the double-lined binary 4755-024, two Gaussian profiles were fitted, with the quoted value being that of the primary.

Heliocentric radial velocities are included in Tables 9-11. The values are plotted for the stars in each cluster in the leftcolumn of Fig. 9; the upper panels show the mean velocity for each star, with the standard deviation $\left(\delta v_{\mathrm{r}}\right)$ of the individual measurements shown in the lower panels. The radial velocity distribution for each cluster is shown in the right-column plots of Fig. 9.

There are a number of specific comments regarding the radial velocity measurements. In the O- and B-type spectra the interstellar $\mathrm{Ca} \mathrm{H} \lambda 3967$ line is easily resolved and was ignored when fitting the $\mathrm{H} \epsilon$ line. In contrast, in the A-type spectra the stellar component of the $\mathrm{CaH}$ line becomes increasingly strong, and the $\mathrm{H} \epsilon$ line was excluded from calculation of the mean velocity. Similarly, as discussed in Sect. 4.7, some stars in NGC 6611 display sharp and strong He I absorption close to the centre of the H8 line; this line was not included in calculation of the mean velocities for any of the NGC 6611 stars. For the G- and K-type spectra in NGC 6611 the quoted velocities are the means of manual measurements of several Fe I lines ( $\lambda \lambda 4071.7,4528.6,4602.9)$ and the $\mathrm{Cr}$ I $\lambda 4254.3$ line.

The mean velocity and its associated dispersion for each cluster is given in Table 8. In calculation of the mean velocities, their dispersion around the mean, and the mean standard deviation of the measurements within each stellar spectrum, it is appropriate to exclude cluster non-members. In NGC 3293 and NGC 4755 we consider stars with spectral types of B8 or later as probable non-members. Given the imposed faint magnitude limit applied in target selection this is reasonable - indeed any B8 or later-type stars observed are often classified as giants and are therefore likely to be non-members of these relatively young clusters (or at least non-coeval with the observed upper main-seqeunce). Following similar arguments in NGC 6611, we do not include stars of type B5 or later.

The mean of the standard deviations of the individual radial velocities was calculated to give an estimate of the expected 

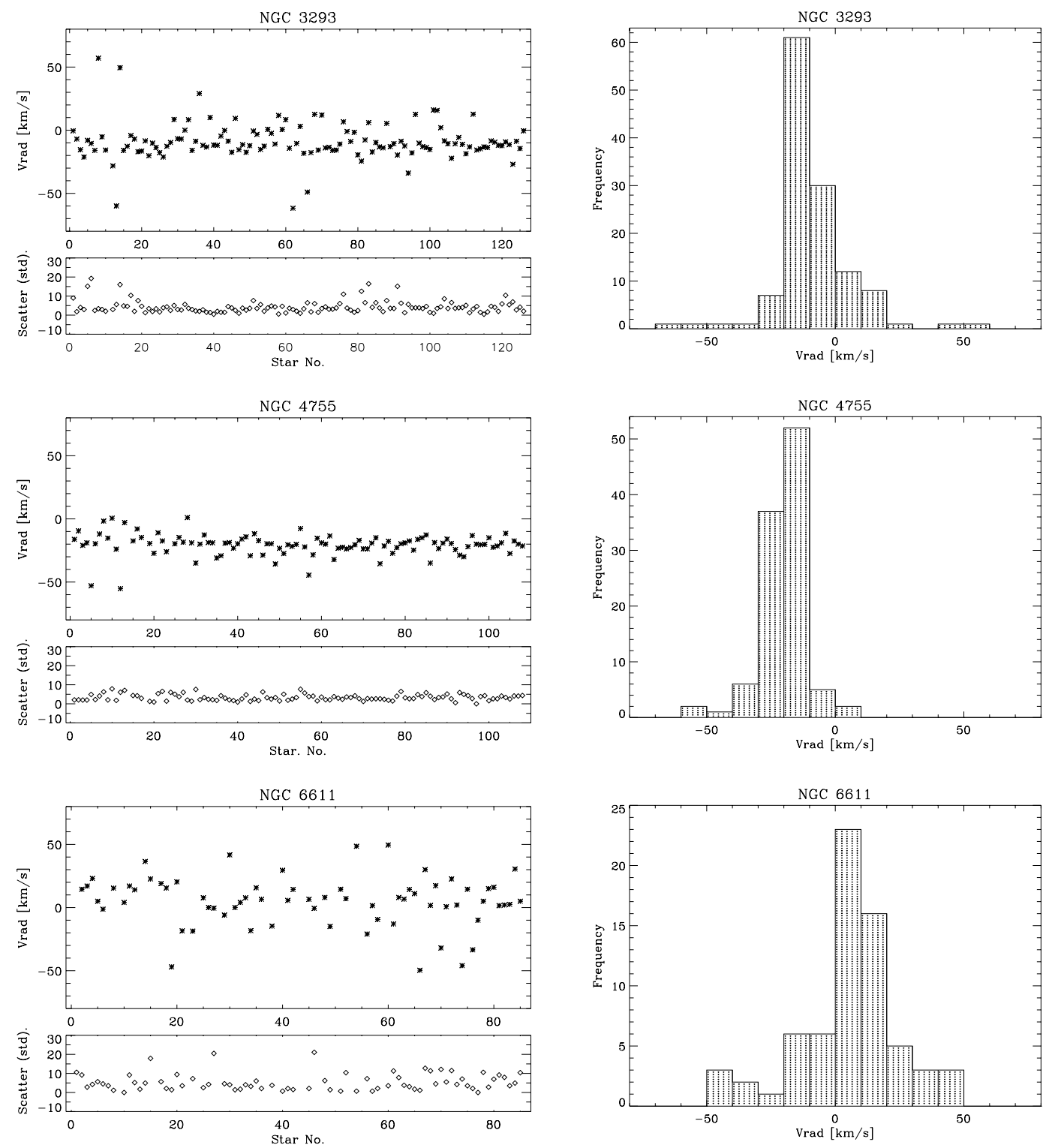

Fig. 9. Distribution of stellar radial velocities. The left-column plots show the radial velocity of each star, with the 1-sigma scatter shown in the lower panel for each cluster. The right-column plots are the binned histograms for each cluster, with a $10 \mathrm{~km} \mathrm{~s}^{-1}$ bin size.

uncertainty (referred to hereafter as $\left.\left\langle\delta v_{\mathrm{r}}\right\rangle\right)$, and this can be directly compared to the velocity dispersion measurements in the clusters to determine if we are measuring statistically significant velocity dispersions. These values (i.e. $\left.\left\langle\delta v_{\mathrm{r}}\right\rangle\right)$, the mean radial velocity of each cluster $\left(\left\langle v_{\mathrm{r}}\right\rangle\right)$ and the dispersion $\left(\sigma_{v}\right)$ are also affected by the stars with variable radial velocities, some of which are the outliers in the plots in Fig. 9. To exclude these we employed a consistent method. The mean $\left(\left\langle v_{\mathrm{r}}\right\rangle\right)$ and standard deviation $\left(\sigma_{v}\right)$ of the velocity distributions were calculated, and any star lying more than $2 \sigma_{v}$ from $\left\langle v_{\mathrm{r}}\right\rangle$ was rejected and the process repeated. This iteration converged quickly and resulted in clear outliers being removed. The same method was used to determine the intrinsic mean error in our analysis $\left\langle\delta v_{\mathrm{r}}\right\rangle$ in each cluster. The values are given in Table 8, together with the final number of stars used, $n$. For each of the clusters the measured velocity dispersion is less than $2\left\langle\delta v_{\mathrm{r}}\right\rangle$, i.e., we do not see any strong evidence for detecting real velocity dispersions. The measurement errors could possibly be reduced using more sophisticated velocity measurement techniques, such as cross-correlation with model spectra, however the rationale for that is beyond the scope of this paper. Note that the mean standard deviation of the individual measurements $\left\langle\delta v_{\mathrm{r}}\right\rangle$ ranges from $3-5 \mathrm{~km} \mathrm{~s}^{-1}$, approximately the onepixel resolution of the spectra. Lastly, significant nebular emission or broad, asymmetric profiles made precise location of the line centre difficult in a number of stars; these are flagged with the usual ":" identifier in the tables to indicate a greater uncertainty $\left(\sim 20 \mathrm{~km} \mathrm{~s}^{-1}\right)$ than for the other values.

Figure 9 shows that there is a tendency for the bright cluster members in NGC 4755 to have a smaller approach velocity $v_{\mathrm{r}}$ than the fainter ones. This was suggested by Feast (1963), although he saw this effect when he combined data from three clusters. We see no evidence in NGC 3293 and NGC 6611 for the effect, however in NGC 4755 the 
$\left\langle v_{\mathrm{r}}\right\rangle$ values for the bright sample (star numbers $\leq 20$, or $V<$ 10 ) is indeed significantly different from the faint sample (star numbers $>20$, or $V>10$ ), see Table 8 . Whether this is physically meaningful remains unclear.

\section{Binarity}

The repeated observations of the NGC 6611 field offer an opportunity to check for single-lined spectroscopic binaries. We find evidence of two stars with companions from this method, 6611-030 and 6611-068. Similarly, the July 18 spectrum of 6611-007 appears to show a relatively sharp-lined early B-type star, however, the July 15 observation reveals it as a double-lined spectroscopic binary. A B0.5 V spectral type is assigned to one component because of weak He II $\lambda 4686$ absorption; the other component is also early B-type, approximately B1.

The two FLAMES observations of 6611-014 display some small profile asymmetries but otherwise appear largely unremarkable. However, inspection of the ISIS spectrum of this star reveals it to be a double-lined binary. From the FLAMES spectra we had initially classified this object as O6 V((f(+))), the suffix indicating the N III emission and evidence of Si IV emission at $\lambda 4116$. From the ISIS spectrum the He I $\lambda 4471$ line of the hotter component appears weaker than for an O6 and we assign a spectral type of $\mathrm{O} 5 \mathrm{~V}((\mathrm{f}(+)))$. The cooler component appears to be that of a late O-type with He I $\lambda 4471$ stronger than the components of the He II lines at $\lambda 4200$ and 4541 . This confirms the suggestion of Bosch et al. (1999) that 6611-014 (W175) comprises two O-type stars (note that a third, relatively low-mass component was reported by Duchêne et al. 2001).

Two further stars in NGC 6611 were identified as binaries by Bosch et al. (1999), namely 6611-001 (W412) and 6611003 (W197). They found that the former is a short period ( $\sim$ days) single-lined binary, but the FEROS and ISIS spectra are in good agreement and thus offer no additional insight. In the case of 6611-003 (initially classified here as 07) Bosch et al. noted the presence of weak Si III absorption that is inconsistent with a mid O-type classification, suggesting a cooler companion. Indeed, shifts of up to $100 \mathrm{~km} \mathrm{~s}^{-1}$ are seen in the Si III lines between the ISIS and FEROS spectra, similarly with the C III blend at $\lambda 4650$. The intensity of the $\lambda 4200$ and $\lambda 4542 \mathrm{He}$ II lines is invariant, but the $\lambda 4686$ line is weaker in the ISIS spectrum, reinforcing the suggestion by Bosch et al. of an approximately B0-type secondary - such a star would contribute to 24686 , but have less of an impact on the other He II lines. The presence of such a secondary could justify revision of the classification of the primary to an earlier type of O6.5, or even O6, depending on the contribution to the He I spectrum by the secondary; therefore we adopt the type employed by Bosch et al. of O6-7 V((f)).

For the remaining 7 stars studied by Bosch et al., our radial velocities are in excellent agreement. However, the comparison of the $\mathrm{H} \alpha$ Balmer line profiles (see Fig. 6) highlights the disagreement between the FLAMES and ISIS spectra of 6611-011 (W314); no differences were revealed between the two FLAMES observations. Closer inspection of the blueregion FLAMES data reveals slightly peculiar profiles for a number of the He I lines, namely $\lambda \lambda 4026,4121,4388$ and 4471, with possibly weak line-doubling seen in the latter two. Both Trumpler (from Walker 1961) and Bosch et al. also suggested possible secondary components. The exact nature of this system is still not entirely clear but the new data here, in particular the $\mathrm{H} \alpha$ profiles in Fig. 6, provide further evidence of a possible companion. That the lines are seen to vary slightly between different epochs may also account for the slightly earlier classification assigned here to the FLAMES-Giraffe spectrum of O9 V (cf. B0 V from Hillenbrand et al. 1993; Bosch et al. 1999).

We have only one observational epoch for NGC 3293 and NGC 4755, but inspection of the FLAMES spectra revealed a double-lined binary, namely 4755-024. The principal component appears as a relatively broad-lined B2.5 dwarf, whilst the relative ratio of the $\mathrm{He}$ I $\lambda 4471$ line to the $\mathrm{Mg}$ II $\lambda 4481$ in the secondary spectrum suggests a spectral type of approximately B5 for the companion.

In comparison to published values, the radial velocities in Sect. 5 also provide an opportunity to detect potential binaries in NGC 3293 and NGC 4755. Feast (1958) published velocities for a number of stars in NGC 3293, 20 of which were observed with FEROS or FLAMES. In general there is reasonable agreement between the two sets of results, but a few stars have significantly discrepant $\left(\left|\Delta v_{\mathrm{r}}\right|>20 \mathrm{~km} \mathrm{~s}^{-1}\right)$ velocities. In NGC 3293 these stars are 3293-008 (F007), 3293-013 (F005), and 3293-014 (F019), all of which were identified by Feast as having variable velocities. Furthermore, Feast suggested that 3293-029 (F028) displayed evidence of line doubling and that it was probably a binary. The FLAMES-Giraffe spectrum of this star displays broad diffuse lines, with some very mild asymmetry in the weaker He I lines such as $\lambda 4144$ and $\lambda 4388$. Its radial velocity $\left(8 \mathrm{~km} \mathrm{~s}^{-1}\right)$ is different to Feast's singular measurement $\left(-13 \mathrm{~km} \mathrm{~s}^{-1}\right)$, but his value was flagged as uncertain and the true nature of this star is still not clear.

In NGC 4755 our results are in good agreement with those of Hernández (1960). Comparison with the results from Feast (1963) again highlights a number of potential binaries, namely, 4755-005 (II-23), 4755-007 (III-05), 4755-012 (III-07), 4755-013 (IV-18) and 4755-021 (I), where the aliases are again taken from Arp \& van Sant (1958). Interestingly Feast presented many measurements for 4755-005, commenting that it is a double-lined binary; our derived velocity of $-55 \mathrm{~km} \mathrm{~s}^{-1}$ is different to those tabulated by Feast, and the spectrum does not appear to contain a secondary component.

\section{Summary}

We have introduced our FLAMES survey of early-type stars in Galactic and Magellanic Cloud open clusters, in particular explaining the philosophy employed in target selection in the Galactic fields. For one of the clusters, NGC 3293, we have reduced the FLAMES data using the pipeline reduction software (girBLDRS) and also using more manual methods in IRAF, finding excellent agreement in the final rectified spectra. Similarly, longslit ISIS spectra of stars in NGC 6611 are also in excellent agreement with the FLAMES data, providing further confidence in the multi-fibre observations.

The spectra were classified, with discussion of specific peculiarities, potential Be-type stars and evidence of binarity. 
In particular, with the benefit of the high-resolution FLAMES spectra we have detected interstellar He I toward some of the NGC 6611 stars, indicative of some highly ionized plasma in the region of the cluster. Stellar radial velocities are given for the majority of our targets, which help to highlight cluster non-members and further potential binaries.

The distributions of spectral types for the three Galactic clusters are summarized in Table 6; in total we have observed 319 Galactic stars. These observations represent a significant increase in the known spectral content of NGC 3293 and NGC 4755, and will serve as comparison stars for our forthcoming data in the Magellanic Clouds.

Acknowledgements. We are extremely grateful to Francesca Primas and the staff at both Paranal and La Silla for their invaluable assistance with the observational programme, and to Andre Blecha and Gilles Simond at the Observatoire de Genève for their helpful responses to our girBLDRS queries. We also thank Nolan Walborn for numerous insightful discussions, Damian Christian and Peter van Hoof for their input on the identification of the non-stellar helium absorption, the referee for their constructive comments, and Cameron Reed for his input on the correct identification of CPD-59 4553 . CJE acknowledges financial support from the UK Particle Physics and Astronomy Research Council (PPARC) under grant PPA/G/S/2001/00131. AH, SSD, and MRV thank the Spanish Ministerio de Educacion y Ciencia under project PNAYA 2001-0436. FN acknowledges support through grants AYA2003-02785E and ESP2002-01627. This paper is based in part on observations made with the Isaac Newton and William Herschel telescopes, operated on the island of La Palma by the Isaac Newton Group in the Spanish Observatorio del Roque de los Muchachos of the Instituto de Astrofísica de Canarias.

\section{Appendix A: Accurate stellar cross-identifications}

In the process of compiling published spectral types for stars in NGC 3293 and NGC 4755, we have discovered several ambiguous cross-identifications in the literature that we attempt to clarify here.

\section{A.1. NGC 3293-012: HD 92007}

Feast (1958) listed (his) star \#6 as HD 92007, with the comment that both this and star \#26 comprise CPD-57 3526 in the Cape Photographic Durchmusterung (CPD). In the SIMBAD database the two stars are sensibly referred to as CPD-57 3526 $(\mathrm{Cl} *$ NGC 3293 FEAS 26, in the SIMBAD notation for the Feast targets) and CPD-57 3526B (Cl* NGC 3293 FEAS 6), but it is the former star (\#26) identified as HD 92007, not the latter. The relevant volume of the Henry Draper Catalogue (Cannon \& Pickering 1919) in fact lists HD 92007 as CPD-5 $7^{\circ} 3527$, (\#27 from Feast); this conclusion is supported from comparisons of our astrometry for Feast \#27 with precessed positions from the HD Catalogue and we adopt this cross-identification. Further confusion is added to the situation by Garrison et al. (1977) who give CPD-57³540 as HD 92007, instead of its correct identification of HD 92044 (Cannon \& Pickering 1919). Curiously, if one follows the relevant links from a SIMBAD query for CPD $-57^{\circ} 3527$, one recovers the MK spectral type for HD 92007 from Hoffleit (1956).

\section{A.2. NGC 3293-003: MCW 1181}

Included in their initial study of southern stars as \#1181, Morgan et al. (1955) classified the spectrum of CD-57³346 as B1 II (where "CD" is the Cordoba Durchmusterung). From the quoted spectral type, one can deduce that Feast (1958) matched MCW 1181 with his star \#22 (CPD-57³506A). The SIMBAD database entry for MCW 1181 reveals cross-references to $\mathrm{CD}-57^{\circ} 3346$ and, more surprisingly, CPD-57 3502 , a well-documented M-type supergiant, classified as M0 Iab (Feast 1958, \#21) and M1.5 Iab-Ib (Morgan \& Keenan 1973). Despite a significant literature search, the explicit source of the CPD and CD cross-references employed by SIMBAD for this star (if one exists) has eluded us, however we note that both Albers (1972) and McGregor \& Hyland (1984) referred to the $\mathrm{M}$-supergiant as $\mathrm{CD}-57^{\circ} 3346$. Here we limit our conclusions to the fact that $\mathrm{CPD}-57^{\circ} 3502$ is not the same source as MCW 1181 and, assuming that the correct star was observed by Feast, therefore not CD $-57^{\circ} 3346$.

To further complicate matters, when the coordinates from the CD catalogue (epoch 1875, available from the Centre de Données astronomiques de Strasbourg, CDS) for CD-57³346 are precessed to epoch 1900, they do not tally with those published by Morgan et al. (1955) for MCW 1181. A similar check for $\mathrm{CD}-57^{\circ} 3340$ (MCW 1179) finds agreement in the positions, suggesting possible typographical or numerical errors in the case of MCW 1181.

\section{A.3. NGC 3293: MCW 1179 and MCW 1182}

The spectra of MCW 1179 (CD-57 3340) and MCW 1182 (CD-57³348) were both classified by Morgan et al. (1955) as B0.5 V. These stars were matched by Feast (1958) as CPD-57 3507 (his \#14) and as CPD-57³500 (\#16), which are 3293-019 and 3293-010 respectively in the current study although as a result of the identical classifications it is not clear which CPD star refers to which MCW target. Again use of the SIMBAD database highlights apparent mismatches. The SIMBAD information for MCW 1179 refers to Feast \#10, and for MCW 1182 to Feast \#22 (the very star to which Feast appears to have attributed the spectral type for MCW 1181). As for MCW 1181, we were again frustrated in our search for the explicit source for the cross-references employed by SIMBAD.

\section{A.4. NGC 4755-008: I-05}

Star I-05 from Arp \& van Sant (1958) was identified as CPD-59 4553 by Hernández (1960). However, SIMBAD matches CPD $-59^{\circ} 4553$ as star R from Feast (1963) and precession of the coordinates in the CPD catalogue leads to similar conclusions. Perry et al. (1976) reported $V=8.86$ for CPD-59 4553 ; a likely explanation is that stars $\mathrm{R}$ and $\mathrm{S}$ were spatially unresolved in their study, i.e. the composite object was the original entry in the CPD catalogue and that the crossidentification of Hernández was incorrect.

\section{References}

Abel, T., Bryan, G. L., \& Norman, M. L. 2002, Science, 295, 93 Albers, H. 1972, ApJ, 176, 623 
Arp, H. C., \& van Sant, C. T. 1958, AJ, 63, 341

Bagnulo, S., Jehin, E., Ledoux, C., et al. 2003, ESO Messenger, 114, 10

Balona, L. A., \& Crampton, D. 1974, MNRAS, 166, 203

Becker, R. H., Fan, X., White, R. L., et al. 2001, AJ, 122, 2850

Belikov, A. N., Kharchenko, N. V., Piskunov, A. E., \& Schilbach, E. 1999, A\&AS, 134, 525

Bidelman, W. P. 1954, PASP, 66, 249

Blaauw, A. 1956, ApJ, 123, 408

Blaauw, A., \& Morgan, W. W. 1953, ApJ, 117, 256

Blecha, A., North, P., Royer, F., \& Simond, G. 2003, BLDR Software - Reference Manual, 1st edn.

Bloom, J. S., Kulkarni, S. R., Price, P. A., et al. 2002, ApJ, 572, L45

Bosch, G. L., Morrell, N. I., \& Niemela, V. S. 1999, Rev. Mex., 35, 85

Bouret, J.-C., Lanz, T., Hillier, D. J., et al. 2003, ApJ, 595, 1182

Bromm, V., Coppi, P. S., \& Larson, R. B. 2002, ApJ, 564, 23

Cannon, A. J., \& Pickering, E. C. 1919, Annals Harvard Obs., 94, 1

Conti, P. S., \& Leep, E. M. 1974, ApJ, 193, 113

Crowther, P. A., Hillier, D. J., Evans, C. J., et al. 2002, ApJ, 579, 774

Dachs, J., \& Kaiser, D. 1984, A\&AS, 58, 411

de Waard, G. J., van Genderen, A. M., \& Bijleveld, W. 1984, A\&AS, 56,373

de Winter, D., Koulis, C., Thé, P. S., et al. 1997, A\&AS, 121, 223

Duchêne, G., Simon, T., Eislöffel, J., \& Bouvier, J. 2001, A\&A, 379, 147

Evans, C. J., \& Howarth, I. D. 2003, MNRAS, 345, 1223

Evans, C. J., Crowther, P. A., Fullerton, A. W., \& Hillier, D. J. 2004a, ApJ, 610, 1021

Evans, C. J., Howarth, I. D., Irwin, M. J., Burnley, A. W., \& Harries, T. J. 2004b, MNRAS, 353, 601

Evans, C. J., Lennon, D. J., Walborn, N. R., Trundle, C., \& Rix, S. A. 2004c, PASP, 116, 909

Feast, M. W. 1958, MNRAS, 118, 618

Feast, M. W. 1963, MNRAS, 126, 11

Feinstein, A., \& Marraco, H. G. 1980, PASP, 92, 266

Galama, T. J., Vreeswijk, P. M., van Paradijs, J., et al. 1998, Nature, 395,670

Garrison, R. F., Hiltner, W. A., \& Schild, R. E. 1977, ApJS, 35, 111

Haehnelt, M. G., Madau, P., Kudritzki, R.-P., \& Haardt, F. 2001, ApJ, 549, L151

Heger, A., \& Langer, N. 2000, ApJ, 544, 1016

Herbig, G. H. 1960, ApJS, 4, 337

Herbig, G. H., \& Dahm, S. E. 2001, PASP, 113, 195

Herbst, W., \& Miller, D. P. 1982, AJ, 87, 1478

Hernández, C. 1960, PASP, 72, 416

Hill, G. M., Walker, G. A. H., \& Yang, S. 1986, PASP, 98, 1186

Hillenbrand, L. A., Massey, P., Strom, S. E., \& Merrill, K. M. 1993, AJ, 106, 1906

Hillier, D. J., Lanz, T., Heap, S. R., et al. 2003, ApJ, 588, 1039

Hiltner, W. A., Garrison, R. F., \& Schild, R. E. 1969, ApJ, 157, 313

Hiltner, W. A., \& Morgan, W. W. 1969, AJ, 74, 1152

Hjorth, J., Sollerman, J., Moller, P., et al. 2003, Nature, 423, 847

Hoffleit, D. 1956, ApJ, 124, 61

Irwin, M. J., \& Lewis, J. 2001, NewAR, 45, 105

Kamp, L. W. 1974, A\&AS, 16, 1

Kaufer, A., Stahl, O., Tubbesing, S., et al. 1999, ESO Messenger, 95, 8

Kudritzki, R.-P. 2002, ApJ, 577, 389

Kudritzki, R.-P., Lennon, D. J., \& Puls, J. 1995, in Science with the VLT, ed. J. R. Walsh, \& I. J. Danziger (Berlin: Springer), 246

Kudritzki, R.-P., Pauldrach, A. W. A., \& Puls, J. 1987, A\&A, 173, 293

Kudritzki, R.-P., Puls, J., Lennon, D. J., et al. 1999, A\&A, 350, 970

Lennon, D. J., Dufton, P. L., \& Fitzsimmons, A. 1992, A\&AS, 94, 569
Lennon, D. J., Mazzali, P. A., Pasian, P., Bonifacio, P., \& Castellani, V. 1993, SSRv, 66, 169

Lesh, J. R. 1968, ApJS, 17, 371

Maeder, A., \& Meynet, G. 2000, A\&A, 361, 101

Maeder, A., \& Meynet, G. 2001, A\&A, 373, 555

Markova, N., Puls, J., Repolust, T., \& Markov, H. 2004, A\&A, 413, 693

McGregor, P. J., \& Hyland, A. R. 1984, ApJ, 277, 149

Merrill, P. W., \& Burwell, C. G. 1949, ApJ, 110, 387

Momany, Y., Vandame, B., Zaggia, S., et al. 2001, A\&A, 379, 436

Morgan, W. W. 1933, ApJ, 77, 330

Morgan, W. W., \& Keenan, P. C. 1973, ARA\&A, 11, 29

Morgan, W. W., Keenan, P. C., \& Kellman, E. 1943, An atlas of stellar spectra (Chicago Univ. Press)

Morgan, W. W., Whitford, A. E., \& Code, A. D. 1953, ApJ, 118, 318

Morgan, W. W., Code, A. D., \& Whitford, A. E. 1955, ApJS, 2, 41

Oudmaijer, R. D., Drew, J. E., Barlow, M. J., Crawford, I. A., \& Proga, D. 1997, MNRAS, 291, 110

Parker, J. W., Garmany, C. D., Massey, P., \& Walborn, N. R. 1992, AJ, 103, 1205

Perry, C. L., Franklin, C. B., Landolt, A. U., \& Crawford, D. L. 1976, AJ, 81, 632

Petrie, R. M. 1965, Publ. Dom. Astrophys. Obs. Victoria, 12, 317

Pettini, M., Ellison, S. L., Bergeron, J., \& Petitjean, P. 2002, A\&A, 391,21

Puls, J., Kudritzki, R.-P., Herrero, A., et al. 1996, A\&A, 305, 171

Repolust, T., Puls, J., \& Herrero, A. 2004, A\&A, 415, 349

Rix, S., Pettini, M., Leitherer, C., et al. 2004, ApJ, 615, 98

Roman, N. G., \& Morgan, W. W. 1950, ApJ, 111, 426

Sanner, J., Brunzendorf, J., Will, J.-M., \& Geffert, M. 2001, A\&A, 369, 511

Schild, R. E. 1970, ApJ, 161, 855

Shapley, A. E., Erb, D. K., Pettini, M., Steidel, C. C., \& Adelberger, K. L. 2004, ApJ, 612, 108

Smartt, S. J., Maund, J. R., Hendry, M. A., et al. 2004, Science, 303, 499

Thé, P. S., de Winter, D., Feinstein, A., \& Westerlund, B. E. 1990, A\&AS, 82, 319

Trundle, C., \& Lennon, D. J. 2005, A\&A, 434, 677

Trundle, C., Lennon, D. J., Puls, J., \& Dufton, P. L. 2004, A\&A, 417, 217

Tucholke, H.-J., Geffert, M., \& Thé, P. S. 1986, A\&AS, 66, 311

Turner, D. G., Grieve, G. R., Herbst, W., \& Harris, W. E. 1980, AJ, 85,1193

Vázquez, G. A., Leitherer, C., Heckman, T. M., et al. 2004, ApJ, 600, 162

Vink, J. S., de Koter, A., \& Lamers, H. J. G. L. M. 2001, A\&A, 369, 574

Walborn, N. R. 1972, AJ, 77, 312

Walborn, N. R. 1973, AJ, 78, 1067

Walborn, N. R. 1976, ApJ, 205, 419

Walborn, N. R. 1982, AJ, 87, 1300

Walborn, N. R. 1983, ApJ, 268, 195

Walborn, N. R., \& Fitzpatrick, E. L. 1990, PASP, 102, 379

Walborn, N. R., Lennon, D. J., Heap, S. R., et al. 2000, PASP, 112, 1243

Walborn, N. R., Morrell, N. I., Howarth, I. D., et al. 2004, ApJ, 608, 1028

Walker, M. F. 1961, ApJ, 133, 438

Welsh, B. Y. 1984, MNRAS, 207, 167

Wilson, O. C. 1937, PASP, 49, 338

Wyithe, J. S. B., \& Loeb, A. 2003, ApJ, 586, 693

Yadav, R. K. S., \& Sagar, R. 2001, MNRAS, 328, 370 


\section{Online Material}




\section{J. Evans et al.: FLAMES survey of massive stars: MW Clusters, Online Material $p 2$}

Table 9. NGC 3293: observational Parameters of Target Stars. The identifications in column two are from: Feast (F, 1958); Turner et al. (T, 1980); Herbst \& Miller (HM, 1982). WFI photometry is quoted for $V>10.75^{\mathrm{m}}$, brighter than this there were saturation problems and values are from Turner et al. and Feinstein \& Marraco (1980). Radial velocities $\left(v_{\mathrm{r}}\right)$ are given in $\mathrm{km} \mathrm{s}^{-1}$. Instrument codes refer to FEROS (F) and Giraffe (G).

\begin{tabular}{|c|c|c|c|c|c|c|c|c|c|}
\hline ID & Alias & $\alpha(2000)$ & $\delta(2000)$ & $V$ & $B-V$ & Sp. type & $v_{\mathrm{r}}$ & Inst. & Comments \\
\hline $3293-001$ & F004 & 103549.33 & -581327.6 & 6.52 & 0.00 & B0 Iab & 0 & $\mathrm{~F}$ & HD 91969, CPD $-57^{\circ} 3508 ; \mathrm{H} \alpha=$ abs + em.(stellar?) \\
\hline 3293-002 & F003 & 103542.02 & -581134.8 & 6.73 & 0.07 & B0.7 Ib & -7 & $\mathrm{~F}$ & HD 91943, CPD-57³499 \\
\hline $3293-003$ & F022 & 103546.57 & -581411.7 & 7.61 & 0.11 & B1 III & -16 & $\mathrm{~F}$ & $\mathrm{CPD}-57^{\circ} 3506 \mathrm{~A}$ \\
\hline 3293-004 & F020 & 103557.72 & -58 1320.6 & 8.03 & 0.02 & B1 III & -21 & $\mathrm{~F}$ & CPD $-57^{\circ} 3523$ \\
\hline 3293-005 & F025 & 103556.55 & -581434.7 & 8.12 & 0.08 & B1 III & -8 & $\mathrm{~F}$ & $\mathrm{CPD}-57^{\circ} 3521$ \\
\hline 3293-006 & F006 & 103558.88 & -581426.1 & 8.21 & 0.07 & B1 III & -10 & $\mathrm{~F}$ & $\mathrm{CPD}-57^{\circ} 3526 \mathrm{~B}$ \\
\hline 3293-007 & F008 & 103616.08 & -581637.9 & 8.25 & 0.18 & B1 III & -16 & $\mathrm{~F}$ & HD 92044, CPD-57³540 \\
\hline 3293-008 & F007 & 103554.22 & -581526.7 & 8.59 & 0.05 & B1 III & 57 & $\mathrm{~F}$ & HD 91983, CPD-57³516 \\
\hline 3293-009 & F048 & 103646.90 & -581753.3 & 8.64 & 0.23 & A7 III & -5 & $\mathrm{~F}$ & HD 92121, CPD-57³563 \\
\hline $3293-010$ & F016 & 103540.72 & -581244.0 & 8.77 & 0.00 & B1 III & -16 & $\mathrm{~F}$ & $\mathrm{CPD}-57^{\circ} 3500$ \\
\hline 3293-011 & F026 & 103558.49 & -581414.8 & 8.85 & 0.04 & Be (B1:) & - & $\mathrm{F}$ & $\mathrm{CPD}-57^{\circ} 3526 ; \mathrm{H} \alpha=$ broad em \\
\hline $3293-012$ & F027 & 103601.60 & -581509.4 & 8.95 & 0.06 & B1 III & -28 & $\mathrm{~F}$ & HD 92007, CPD-57³527 \\
\hline $3293-013$ & F005 & 103608.34 & -581304.1 & 9.03 & -0.04 & B1 III & -60 & $\mathrm{~F}$ & HD 92024, CPD-57³533 \\
\hline 3293-014 & F019 & 103558.63 & -581232.1 & 9.09 & 0.19 & B0.5 IIIn & 50 & $\mathrm{~F}$ & $\mathrm{CPD}-57^{\circ} 3524 \mathrm{~A}$ \\
\hline 3293-015 & $\mathrm{T} 133$ & 103554.92 & -581258.9 & 9.11 & -0.01 & $\mathrm{~B} 1 \mathrm{~V}$ & -16 & $\mathrm{~F}$ & $\mathrm{CPD}-57^{\circ} 3515$ \\
\hline $3293-016$ & F023 & 103548.45 & -581414.5 & 9.21 & 0.03 & $\mathrm{~B} 2.5 \mathrm{~V}$ & -14 & $\mathrm{G}$ & CPD $-57^{\circ} 3506 \mathrm{~B}$; strongly blended \\
\hline 3293-017 & F024 & 103553.65 & -581447.6 & 9.22 & 0.04 & B1 V & -4 & $\mathrm{~F}$ & $\mathrm{CPD}-57^{\circ} 3517$ \\
\hline 3293-018 & F018 & 103557.81 & -581221.1 & 9.26 & -0.04 & B1 V & -7 & $\mathrm{~F}$ & $\mathrm{CPD}-57^{\circ} 3524 \mathrm{~B}$ \\
\hline 3293-019 & F014 & 103548.22 & -581232.9 & 9.27 & -0.04 & B1 V & -17 & $\mathrm{~F}$ & $\mathrm{CPD}-57^{\circ} 3507$ \\
\hline $3293-020$ & F010 & 103530.07 & -581208.0 & 9.55 & 0.03 & B1.5 III & -16 & G & HDE 303067 \\
\hline $3293-021$ & T065 & 103543.31 & -581333.4 & 9.85 & 0.03 & B1.5 III & -8 & $\mathrm{G}$ & $\mathrm{CPD}-57^{\circ} 3503$ \\
\hline $3293-022$ & F012 & 103532.30 & -581522.0 & 9.97 & 0.12 & $\mathrm{Be}(\mathrm{B} 0.5-1.5 \mathrm{n})$ & -20 & $\mathrm{G}$ & HDE $303075 ; \mathrm{H} \alpha=$ twin \\
\hline $3293-023$ & $\mathrm{~T} 131$ & 103555.39 & -58 1219.7 & 10.01 & -0.05 & B 1.5 III & -10 & $\mathrm{G}$ & $\mathrm{CPD}-57^{\circ} 3519$ \\
\hline $3293-024$ & F009 & 103604.91 & -581043.3 & 10.01 & -0.01 & B1.5 III & -14 & G & HDE 303065 \\
\hline 3293-025 & F015 & 103545.17 & -581223.6 & 10.01 & 0.00 & B2 III & $-18:$ & $\mathrm{G}$ & CPD-57 3504 ; strongly blended \\
\hline 3293-026 & F013 & 103556.60 & -581131.4 & 10.16 & -0.02 & B2 III & -21 & G & $\mathrm{CPD}-57^{\circ} 3522$ \\
\hline $3293-027$ & T079 & 103539.97 & -581356.9 & 10.22 & 0.07 & $\mathrm{Be}(\mathrm{B} 0.5-1.5 \mathrm{n})$ & -13 & $\mathrm{G}$ & $\mathrm{H} \alpha=\mathrm{abs}+\mathrm{twin}$ \\
\hline $3293-028$ & $\mathrm{~T} 132$ & 103556.61 & -581240.7 & 10.26 & 0.01 & $\mathrm{~B} 2 \mathrm{~V}$ & -10 & $\mathrm{G}$ & $\mathrm{CPD}-57^{\circ} 3520$ \\
\hline 3293-029 & F028 & 103605.95 & -581427.0 & 10.32 & -0.01 & B0.5-B1.5 Vn & 8 & $\mathrm{G}$ & $\mathrm{CPD}-57^{\circ} 3531$ \\
\hline $3293-030$ & F017 & 103553.01 & -581216.8 & 10.51 & -0.04 & $\mathrm{~B} 2 \mathrm{~V}$ & -7 & $\mathrm{G}$ & $\mathrm{CPD}-57^{\circ} 3514$ \\
\hline 3293-031 & F033 & 103603.51 & -581440.0 & 10.66 & 0.05 & $\mathrm{~B} 2 \mathrm{~V}$ & -7 & G & $\mathrm{CPD}-57^{\circ} 3528$ \\
\hline $3293-032$ & F029 & 103554.67 & -581348.6 & 10.69 & 0.01 & B0.5-B1.5 Vn & 0 & G & $\mathrm{CPD}-57^{\circ} 3518$ \\
\hline $3293-033$ & F041 & 103433.88 & -581228.2 & 10.72 & 0.66 & B8 III & 8 & $\mathrm{G}$ & HDE 303073 \\
\hline 3293-034 & T086 & 103549.01 & -581454.1 & 10.74 & 0.11 & B2 IIIh & -16 & $\mathrm{G}$ & \\
\hline $3293-035$ & F045 & 103640.29 & -580554.7 & 10.81 & 0.09 & B2 V & -9 & $\mathrm{G}$ & HDE 303062 \\
\hline $3293-036$ & F036 & 103617.02 & -580826.5 & 10.90 & 0.19 & A5 III & 29: & $\mathrm{G}$ & HDE 303066 \\
\hline $3293-037$ & T089 & 103607.64 & -581520.2 & 10.94 & 0.04 & B2 V & -12 : & G & \\
\hline 3293-038 & - & 103506.64 & -58 1034.7 & 11.00 & -0.04 & $\mathrm{~B} 2.5 \mathrm{~V}$ & $-13:$ & $\mathrm{G}$ & \\
\hline 3293-039 & F051 & 103704.06 & -580801.3 & 11.07 & 0.07 & A3 III & 10 & $\mathrm{G}$ & HDE 303063 \\
\hline $3293-040$ & T063 & 103544.05 & -581345.9 & 11.21 & 0.05 & $\mathrm{Be}(\mathrm{B} 3 \mathrm{n})$ & -12 & $\mathrm{G}$ & $\mathrm{H} \alpha=\mathrm{abs}+\mathrm{twin}$ \\
\hline $3293-041$ & T087 & 103544.66 & -581430.1 & 11.22 & 0.04 & B2.5 V & -12 & $\mathrm{G}$ & \\
\hline $3293-042$ & F044 & 103558.93 & -580517.8 & 11.27 & 0.11 & A3 III & -5 & G & HDE 303061 \\
\hline $3293-043$ & T092 & 103528.51 & -581249.6 & 11.32 & 0.07 & B3 V & 0 & $\mathrm{G}$ & \\
\hline 3293-044 & F039 & 103438.31 & -580742.3 & 11.35 & 0.10 & A3 III & $-9:$ & G & HDE 303071 \\
\hline $3293-045$ & - & 103613.70 & -581732.7 & 11.42 & 0.32 & $\mathrm{Be}(\mathrm{B} 1-2 \mathrm{n})$ & -18 & $\mathrm{G}$ & $\mathrm{H} \alpha=$ twin \\
\hline $3293-046$ & F046 & 103515.05 & -580426.8 & 11.44 & 0.11 & A7 III & 9 & $\mathrm{G}$ & HDE 303060 \\
\hline $3293-047$ & HM322 & 103536.62 & -581604.0 & 11.55 & 0.13 & B $2.5 \mathrm{~V}$ & -16 & $\mathrm{G}$ & \\
\hline $3293-048$ & HM292 & 103544.99 & -581635.0 & 11.56 & 0.16 & $\mathrm{~B} 2.5 \mathrm{~V}$ & -12 & G & \\
\hline $3293-049$ & - & 103420.79 & -581330.5 & 11.64 & 0.07 & B2.5 V & -18 & $\mathrm{G}$ & \\
\hline $3293-050$ & T075 & 103541.94 & -581156.7 & 11.69 & 0.05 & B3 Vn & -12 & $\mathrm{G}$ & \\
\hline
\end{tabular}


Table 9. continued.

\begin{tabular}{|c|c|c|c|c|c|c|c|c|c|}
\hline ID & Alias & $\alpha(2000)$ & $\delta(2000)$ & $V$ & $B-V$ & Sp. type & $v_{\mathrm{r}}$ & Inst. & Comments \\
\hline $3293-051$ & - & 103438.85 & -580729.7 & 11.73 & 0.20 & A5 III & -1 & $\mathrm{G}$ & \\
\hline $3293-052$ & HM459 & 103529.91 & -580928.3 & 11.77 & 0.22 & A3 III & -3 & G & \\
\hline $3293-053$ & $\mathrm{~T} 130$ & 103550.74 & -581141.2 & 11.83 & 0.03 & B3 V & -15 & G & \\
\hline $3293-054$ & - & 103625.86 & -581436.2 & 11.85 & 0.06 & A0 III & -13 & G & \\
\hline $3293-055$ & HM212 & 103628.66 & -581335.5 & 11.88 & 0.12 & A3 III & 1 & G & \\
\hline $3293-056$ & - & 103513.55 & -581112.4 & 11.91 & 0.00 & B3 V & -2 & G & \\
\hline $3293-057$ & T084 & 103545.30 & -581528.0 & 11.92 & 0.21 & B3 V & -11 & G & faint companion in WFI image \\
\hline 3293-058 & - & 103538.86 & -580426.3 & 11.99 & 0.08 & A0 IIp (Si) & 12 & G & \\
\hline 3293-059 & HM277 & 103606.57 & -581753.8 & 12.00 & 0.29 & B5 III-Vn & 0 & G & \\
\hline $3293-060$ & HM231 & 103521.10 & -581200.5 & 12.02 & 0.07 & A2 III & 8 & G & \\
\hline $3293-061$ & T052 & 103554.72 & -581237.0 & 12.03 & 0.05 & B5 V & -14 & G & \\
\hline $3293-062$ & HM264 & 103605.25 & -581645.5 & 12.03 & 0.15 & B3 V & -62 & G & \\
\hline $3293-063$ & - & 103704.82 & -581008.1 & 12.05 & 0.09 & B5 V & -10 & G & \\
\hline $3293-064$ & - & 103554.40 & -581256.3 & 12.06 & 0.06 & A0 II & 3 & G & adjacent to $\mathrm{T} 133$ \\
\hline $3293-065$ & - & 103442.02 & -581541.9 & 12.06 & 0.14 & B5 III-V & -18 & G & \\
\hline $3293-066$ & T090 & 103557.12 & -581521.6 & 12.07 & 0.16 & B5 V & -49 : & G & \\
\hline $3293-067$ & $\mathrm{~T} 117$ & 103613.48 & -581120.7 & 12.11 & 0.32 & B3 V & $-18:$ & G & \\
\hline 3293-068 & - & 103509.57 & -580747.7 & 12.21 & 0.06 & B9 III & 12 & G & \\
\hline 3293-069 & HM393 & 103524.10 & -581328.4 & 12.22 & 0.07 & B5 V & -16 & G & \\
\hline $3293-070$ & - & 103454.80 & -580723.3 & 12.22 & 0.14 & B5 III-V & 12 & G & \\
\hline $3293-071$ & - & 103448.62 & -58 1313.4 & 12.23 & 0.22 & A7 II-III & -14 & G & \\
\hline $3293-072$ & HM408 & 103516.11 & -581259.7 & 12.25 & 0.00 & B8 IIp & -14 & G & composite spectrum? \\
\hline $3293-073$ & - & 103538.51 & -581306.8 & 12.25 & 0.11 & B6-7 V & -16 & G & \\
\hline $3293-074$ & HM403 & 103521.96 & -581253.6 & 12.28 & 0.09 & B8 III & $-16:$ & G & \\
\hline $3293-075$ & HM299 & 103522.72 & -581708.1 & 12.29 & 0.12 & B5 IIIn & -11 & G & \\
\hline $3293-076$ & - & 103502.75 & -581303.4 & 12.29 & 0.34 & F0 III & 7 & G & \\
\hline $3293-077$ & - & 103458.30 & -581118.6 & 12.32 & 0.20 & B6-7 V & -1 & G & no $\lambda 4656,6515$ data \\
\hline 3293-078 & - & 103607.04 & -580525.6 & 12.36 & 0.13 & A3 III & -9 & G & \\
\hline 3293-079 & HM352 & 103523.68 & -581506.1 & 12.36 & 0.13 & A0 III & -2 & G & \\
\hline $3293-080$ & - & 103606.56 & -580655.1 & 12.36 & 0.14 & B5 V & -20 & G & \\
\hline $3293-081$ & - & 103645.39 & -580950.5 & 12.38 & 0.32 & A5 II & $-24:$ & G & \\
\hline $3293-082$ & - & 103547.53 & $\begin{array}{llll}-58 & 05 & 13.3\end{array}$ & 12.40 & 0.21 & B5 III & $-8:$ & G & \\
\hline $3293-083$ & - & 103633.50 & -582033.8 & 12.46 & 0.28 & A3 III & 6: & G & \\
\hline $3293-084$ & T093 & 103602.29 & -581258.1 & 12.48 & 0.11 & B5 V & -17 & G & \\
\hline $3293-085$ & - & 103628.00 & -580818.0 & 12.49 & 0.13 & B5 V & -10 & G & \\
\hline $3293-086$ & - & 103554.92 & -580649.3 & 12.52 & 0.16 & B5 V & $-13:$ & G & \\
\hline $3293-087$ & - & 103504.74 & -581415.4 & 12.60 & 0.04 & B5 V & -14 & G & \\
\hline 3293-088 & HM401 & 103521.74 & -581237.8 & 12.60 & 0.24 & F0 III-V & 5 & G & \\
\hline 3293-089 & - & 103650.05 & -580452.3 & 12.64 & 0.15 & B8 III & -13 & G & \\
\hline $3293-090$ & T104 & 103545.81 & -581600.0 & 12.67 & 0.31 & B6-7 V & -11 & G & \\
\hline 3293-091 & - & 103559.03 & -580553.7 & 12.68 & 0.34 & A7 II & -20 & G & \\
\hline $3293-092$ & - & 103615.02 & -580804.5 & 12.70 & 0.16 & B9 III & -9 & G & \\
\hline $3293-093$ & T081 & 103535.68 & -581356.4 & 12.71 & 0.14 & B6-7 V & -12 & G & \\
\hline 3293-094 & $\mathrm{T} 121$ & 103617.91 & -581429.5 & 12.71 & 0.20 & B5 V & $-34:$ & G & \\
\hline 3293-095 & $\mathrm{T} 120$ & 103612.90 & -581324.9 & 12.72 & 0.13 & B6-7 V & -18 & G & \\
\hline $3293-096$ & - & 103503.25 & -581426.8 & 12.74 & 0.23 & B6-7 III & 12: & G & \\
\hline $3293-097$ & $\mathrm{~T} 124$ & 103616.92 & -581500.0 & 12.75 & 0.13 & B6-7 III & -10 & G & faint companion in WFI image \\
\hline 3293-098 & T055 & 103552.82 & -581311.7 & 12.75 & 0.14 & B8 III-V & -13 & G & \\
\hline 3293-099 & - & 103646.36 & -580929.1 & 12.78 & 0.18 & B5 Vn & -14 & G & \\
\hline $3293-100$ & HM313 & 103551.31 & -581617.8 & 12.81 & 0.14 & B6-7 III-Vn & $-15:$ & G & \\
\hline
\end{tabular}


C. J. Evans et al.: FLAMES survey of massive stars: MW Clusters, Online Material $p 4$

Table 9. continued.

\begin{tabular}{llcccccrll}
\hline \hline ID & Alias & $\alpha(2000)$ & $\delta(2000)$ & $V$ & $B-V$ & Sp. type & $v_{\text {r }}$ & Inst. & Comments \\
\hline $3293-101$ & - & 103647.50 & -581801.2 & 12.82 & 0.36 & F0 III & 16 & G & \\
$3293-102$ & - & 103453.56 & -582058.7 & 12.83 & 0.28 & A2 III & 16 & G & \\
$3293-103$ & - & 103457.00 & -580806.0 & 12.83 & 0.29 & A7 II & 2 & G & \\
$3293-104$ & T069 & 103540.29 & -581301.0 & 12.88 & 0.11 & B6-7 V & $-8:$ & G & \\
$3293-105$ & - & 103557.85 & -581502.8 & 12.89 & 0.19 & B8 III-V & -11 & G & \\
$3293-106$ & F034 & 103550.51 & -581212.6 & 12.90 & 0.08 & B6-7 V & -22 & G & \\
$3293-107$ & HM243 & 103610.82 & -581548.9 & 12.91 & 0.22 & B8 III-V & -11 & G & \\
$3293-108$ & - & 103609.85 & -580544.3 & 12.92 & 0.13 & B6-7 V & -6 & G & \\
$3293-109$ & HM454 & 103532.89 & -581016.2 & 12.92 & 0.15 & B5 V & -11 & G & \\
$3293-110$ & - & 103702.26 & -581237.1 & 12.93 & 0.28 & A3 II & -19 & G & \\
$3293-111$ & T091 & 103555.41 & -581537.1 & 12.96 & 0.16 & B6-7 III-V & -13 & G & \\
$3293-112$ & - & 103434.76 & -581605.0 & 12.99 & 0.18 & A5 III & $13:$ & G & \\
$3293-113$ & T100 & 103558.37 & -581440.9 & 13.04 & 0.14 & B6-7 V & -16 & G & \\
$3293-114$ & - & 103638.81 & -581357.4 & 13.07 & 0.25 & B6-7 III-V & -15 & G & \\
$3293-115$ & - & 103553.98 & -581504.4 & 13.11 & 0.14 & B8 III-V & -13 & G & \\
$3293-116$ & T071 & 103535.84 & -581307.6 & 13.12 & 0.11 & B6-7 V & -14 & G & \\
$3293-117$ & T114 & 103609.76 & -581057.8 & 13.15 & 0.14 & B8 III-V & -8 & G & \\
$3293-118$ & - & 103506.71 & -580507.7 & 13.17 & 0.06 & B8 III-V & -10 & G & \\
$3293-119$ & T109 & 103536.70 & -581528.2 & 13.18 & 0.22 & B9 III & -12 & G & \\
$3293-120$ & - & 103447.74 & -580727.4 & 13.20 & 0.33 & B5 V & $-12:$ & G & \\
$3293-121$ & HM316 & 103541.32 & -581539.2 & 13.23 & 0.21 & B8: III & -9 & G & \\
$3293-122$ & T119 & 103618.64 & -581253.5 & 13.24 & 0.15 & B9 III & -12 & G & \\
$3293-123$ & - & 103553.12 & -581426.0 & 13.26 & 0.20 & B8 III & -27 & G & \\
$3293-124$ & - & 103544.93 & -580511.2 & 13.28 & 0.21 & B8 III & -9 & G & \\
$3293-125$ & T067 & 103547.53 & -581246.9 & 13.29 & 0.10 & B8 III-V & $-14:$ & G & \\
$3293-126$ & HM285 & 103530.63 & -581740.4 & 13.29 & 0.22 & A7 III & 0 & G & \\
\hline
\end{tabular}


Table 10. NGC 4755: observational Parameters of Target Stars. The identifications in column two are those of Arp \& van Sant (1958); uncertain matches from the finding charts are denoted by “*”. WFI photometry is quoted for $V>11.5^{\mathrm{m}}$, brighter than this there were saturation problems and values are from Sanner et al. (2001). Radial velocities $\left(v_{\mathrm{r}}\right)$ are given in $\mathrm{km} \mathrm{s}^{-1}$. Instrument codes refer to FEROS (F) and Giraffe (G).

\begin{tabular}{|c|c|c|c|c|c|c|c|c|c|}
\hline ID & Alias & $\alpha(2000)$ & $\delta(2000)$ & $V$ & $B-V$ & Sp. type & $v_{\mathrm{r}}$ & Inst. & Comments \\
\hline $4755-001$ & A & 125321.92 & -601947.4 & 5.77 & 0.32 & B9 Ia & -16 & $\mathrm{~F}$ & HD 111904, CPD-594529 \\
\hline 4755-002 & $\mathrm{B}$ & 125348.94 & -602236.8 & 5.98 & 0.22 & B3 Ia & -10 & $\mathrm{~F}$ & $\kappa$ Crucis; HD 111973, CPD-59 4555; H $\alpha=$ broad em \\
\hline $4755-003$ & $\mathrm{C}$ & 125359.81 & -60208.7 & 6.80 & 0.24 & B2 III & -21 & $\mathrm{~F}$ & HD 111990, CPD $-59^{\circ} 4566 ; \mathrm{H} \alpha=\mathrm{abs}+\mathrm{em}$ (stellar?) \\
\hline $4755-004$ & $\mathrm{I}-06$ & 125337.64 & -602125.7 & 6.92 & 0.20 & $\mathrm{~B} 1.5 \mathrm{Ib}$ & -19 & $\mathrm{~F}$ & HD $111934, \mathrm{CPD}-59^{\circ} 4543$ \\
\hline $4755-005$ & II-23 & 125347.30 & -601955.6 & 7.96 & 0.20 & B2 III & -53 & $\mathrm{~F}$ & $\mathrm{CPD}-59^{\circ} 4551$ \\
\hline 4755-006 & $\mathrm{E}$ & 125346.52 & -602412.6 & 8.37 & 0.14 & B1 III & -20 & $\mathrm{~F}$ & $\mathrm{CPD}-59^{\circ} 4552$ \\
\hline $4755-007$ & III-05 & 125349.52 & -60233.1 & 8.58 & 0.11 & $\mathrm{~B} 1 \mathrm{~V}$ & -12 & $\mathrm{~F}$ & $\mathrm{CPD}-59^{\circ} 4557$ \\
\hline $4755-008$ & $\mathrm{I}-05$ & 125339.20 & -602113.0 & 8.66 & 0.14 & B $0.5 \mathrm{~V}$ & -2 & $\mathrm{~F}$ & \\
\hline 4755-009 & $\mathrm{F}$ & 125357.58 & -602458.3 & 9.01 & 0.11 & B1 V & -15 & $\mathrm{~F}$ & $\mathrm{CPD}-59^{\circ} 4564$ \\
\hline $4755-010$ & II-01 & 125352.03 & -602216.0 & 9.38 & 0.16 & $\mathrm{~B} 1 \mathrm{~V}$ & 0 & $\mathrm{~F}$ & \\
\hline $4755-011$ & $\mathrm{R}$ & 125346.57 & -602218.5 & 9.58 & 0.17 & B $1.5 \mathrm{~V}$ & -24 & $\mathrm{~F}$ & \\
\hline 4755-012 & III-07 & 125352.27 & -602228.0 & 9.60 & 0.16 & $\mathrm{~B} 1.5 \mathrm{~V}$ & -55 & $\mathrm{~F}$ & $\mathrm{CPD}-59^{\circ} 4560$ \\
\hline $4755-013$ & IV -18 & 125335.52 & -602346.9 & 9.68 & 0.15 & $\mathrm{~B} 1.5 \mathrm{~V}$ & -3 & $\mathrm{~F}$ & $\mathrm{CPD}-59^{\circ} 4542$ \\
\hline $4755-014$ & $\mathrm{~S}$ & 125347.28 & -602220.2 & 9.72 & 0.20 & Be (B1:) & - & $\mathrm{F}$ & $\mathrm{H} \alpha=$ twin \\
\hline $4755-015$ & G & 125320.69 & -602317.1 & 9.74 & 0.16 & B1 V & -18 & $\mathrm{~F}$ & CPD $-59^{\circ} 4528$ \\
\hline $4755-016$ & III-01 & 125343.91 & -602229.5 & 9.76 & 0.12 & $\mathrm{~B} 1.5 \mathrm{~V}$ & -8 & $\mathrm{~F}$ & $\mathrm{CPD}-59^{\circ} 4549$ \\
\hline $4755-017$ & $\mathrm{H}$ & 125333.27 & -602433.5 & 9.90 & 0.18 & $\mathrm{~B} 1.5 \mathrm{~V}$ & -15 & $\mathrm{~F}$ & $\mathrm{CPD}-59^{\circ} 4540$ \\
\hline $4755-018$ & IV -17 & 125339.02 & -602343.8 & 9.96 & 0.16 & Be (B1.5:) & - & $\mathrm{F}$ & $\mathrm{CPD}-59^{\circ} 4546 ; \mathrm{H} \alpha=$ broad em. \\
\hline 4755-019 & IV -52 & 125310.23 & -602559.8 & 10.04 & 0.17 & $\mathrm{~B} 1.5 \mathrm{~V}$ & -20 & $\mathrm{~F}$ & HDE $312079, \mathrm{CPD}-59^{\circ} 4523$ \\
\hline $4755-020$ & IV -54 & 125314.25 & -602738.9 & 10.05 & 0.13 & $\mathrm{~B} 2 \mathrm{~V}$ & -27 & $\mathrm{G}$ & \\
\hline $4755-021$ & I & 125347.02 & -601835.7 & 10.29 & 0.20 & $\mathrm{~B} 1.5 \mathrm{Vn}$ & -11 & $\mathrm{~F}$ & $\mathrm{CPD}-59^{\circ} 4550$ \\
\hline $4755-022$ & $\mathrm{I}-17$ & 125324.28 & -602131.0 & 10.89 & 0.12 & $\mathrm{~B} 2.5 \mathrm{Vn}$ & -18 & $\mathrm{G}$ & $\mathrm{CPD}-59^{\circ} 4531$ \\
\hline $4755-023$ & $\mathrm{~J}$ & 125325.96 & -602048.1 & 10.99 & 0.18 & $\mathrm{~B} 2 \mathrm{~V}$ & -26 & G & $\mathrm{CPD}-59^{\circ} 4537$ \\
\hline $4755-024$ & III-32 & 125356.66 & -602632.9 & 11.00 & 0.12 & $\mathrm{~B} 2.5 \mathrm{~V}+\mathrm{B} 5:$ & -85 & G & $\mathrm{CPD}-59^{\circ} 4563$ \\
\hline $4755-025$ & III-26 & 125403.63 & -602521.1 & 11.02 & 0.14 & B $2.5 \mathrm{~V}$ & -20 & G & \\
\hline $4755-026$ & IV -03 & 125339.41 & -602240.0 & 11.19 & 0.24 & $\mathrm{~B} 2.5 \mathrm{~V}$ & -15 & G & \\
\hline $4755-027$ & II-14 & 125343.22 & -602047.5 & 11.21 & 0.17 & $\mathrm{~B} 2.5 \mathrm{Vn}$ & -18 & $\mathrm{G}$ & \\
\hline $4755-028$ & $\mathrm{I}-50$ & 125325.39 & -601618.3 & 11.22 & 0.31 & B9 III & 1 & G & \\
\hline 4755-029 & $\mathrm{I}-38$ & 125325.43 & -601911.5 & 11.23 & 0.19 & $\mathrm{~B} 2 \mathrm{~V}$ & -19 & G & $\mathrm{CPD}-59^{\circ} 4536$ \\
\hline $4755-030$ & II-20 & 125408.61 & -602138.7 & 11.31 & 0.21 & $\mathrm{~B} 2.5 \mathrm{Vn}$ & -35 & G & \\
\hline $4755-031$ & IV -59 & 125250.69 & -602536.9 & 11.34 & 0.24 & $\mathrm{~B} 2.5 \mathrm{~V}$ & -20 & $\mathrm{G}$ & \\
\hline $4755-032$ & IV -26 & 125309.90 & -602227.5 & 11.35 & 0.19 & $\mathrm{~B} 2.5 \mathrm{~V}$ & -13 & G & \\
\hline $4755-033$ & IV -11 & 125339.95 & -602327.1 & 11.35 & 0.24 & B3 V & -19 & $\mathrm{G}$ & \\
\hline 4755-034 & $\mathrm{K}$ & 125322.61 & -602347.8 & 11.39 & 0.23 & B3 V & -19 & $\mathrm{G}$ & $\mathrm{CPD}-59^{\circ} 4530$ \\
\hline $4755-035$ & $\mathrm{I}-07$ & 125338.21 & -602145.3 & 11.41 & 0.23 & B5 V & -31 & $\mathrm{G}$ & \\
\hline $4755-036$ & III-16 & 125343.36 & -602402.5 & 11.45 & 0.16 & B3 Vn & -29 & G & \\
\hline $4755-037$ & III-18 & 125347.01 & -602518.4 & 11.48 & 0.13 & $\mathrm{~B} 2.5 \mathrm{~V}$ & -19 & $\mathrm{G}$ & \\
\hline $4755-038$ & III-13 & 125351.83 & -602354.5 & 11.54 & 0.16 & B3 Ve & -19 & $\mathrm{G}$ & $\mathrm{H} \alpha=\mathrm{v}$. weak, twin \\
\hline $4755-039$ & IV -06 & 125337.13 & -602255.4 & 11.54 & 0.16 & $\mathrm{~B} 2.5 \mathrm{~V}$ & -23 & $\mathrm{G}$ & \\
\hline $4755-040$ & - & 125422.49 & -601314.4 & 11.54 & 0.32 & $\mathrm{~B} 2.5 \mathrm{~V}$ & -20 & G & \\
\hline $4755-041$ & II-13 & 125344.05 & -602058.1 & 11.58 & 0.21 & B3 V & -16 & G & \\
\hline $4755-042$ & I-39 & 125326.48 & -601900.5 & 11.58 & 0.29 & B3 Vn & -14 & G & \\
\hline $4755-043$ & II- 15 & 125349.41 & -602057.6 & 11.59 & 0.22 & B3 V & -29 & G & \\
\hline $4755-044$ & - & 125444.34 & -602514.2 & 11.59 & 0.28 & B5 V & -12 & $\mathrm{G}$ & \\
\hline $4755-045$ & IV -08 & 125332.47 & -602239.3 & 11.61 & 0.22 & B3 V & -17 & $\mathrm{G}$ & \\
\hline $4755-046$ & - & 125407.16 & -602313.4 & 11.62 & 0.20 & B3 Vn & $-29:$ & G & \\
\hline $4755-047$ & IV -13 & 125326.20 & -602252.2 & 11.62 & 0.23 & B3 V & -20 & G & \\
\hline $4755-048$ & IV -47 & 125309.66 & -602413.7 & 11.63 & 0.19 & B3 V & -20 & $\mathrm{G}$ & \\
\hline $4755-049$ & - & 125254.70 & -601516.4 & 11.65 & 0.34 & B5 V & $-36:$ & G & \\
\hline $4755-050$ & IV -53 & 125316.55 & -602611.7 & 11.65 & 0.36 & A2 IIe & -23 & $\mathrm{G}$ & $\mathrm{H} \alpha=\mathrm{abs}+\mathrm{twin}$ \\
\hline
\end{tabular}


Table 10. continued.

\begin{tabular}{|c|c|c|c|c|c|c|c|c|c|}
\hline ID & Alias & $\alpha(2000)$ & $\delta(2000)$ & $V$ & $B-V$ & Sp. type & $v_{\mathrm{r}}$ & Inst. & Comments \\
\hline $4755-051$ & - & 125353.09 & -602307.4 & 11.75 & 0.23 & B3 Vn & -28 & $\bar{G}$ & \\
\hline $4755-052$ & IV -42 & 125333.93 & -602622.4 & 11.77 & 0.17 & B3 V & -20 & G & \\
\hline $4755-053$ & IV-27 & 125341.83 & -602438.4 & 11.78 & 0.18 & B3 Vn & -22 & G & \\
\hline $4755-054$ & IV -46 & 125314.03 & -602413.4 & 11.78 & 0.19 & B3 V & -20 & G & \\
\hline $4755-055$ & - & 125432.49 & -602601.4 & 11.83 & 0.45 & A7 II & $-8:$ & G & \\
\hline $4755-056$ & - & 125329.35 & -602119.5 & 11.86 & 0.29 & B3 Vn & -22 & G & blended in WFI image \\
\hline $4755-057$ & II-38 & 125432.18 & -601624.9 & 11.94 & 0.36 & B6-7 III-Ve & $-44:$ & G & $\mathrm{H} \alpha=\mathrm{abs}+\mathrm{twin}$ \\
\hline $4755-058$ & II-07 & 125345.46 & -602108.3 & 12.07 & 0.26 & B3 Vn & -28 & G & \\
\hline $4755-059$ & - & 125436.24 & -601923.4 & 12.10 & 0.52 & F0 III & -15 & G & \\
\hline $4755-060$ & IV -33 & 125336.92 & -602527.4 & 12.12 & 0.19 & B5 V & -19 & G & \\
\hline $4755-061$ & $\mathrm{I}-22$ & 125308.94 & -602122.7 & 12.15 & 0.25 & B5 V & -20 & G & strong Si II for type \\
\hline $4755-062$ & IV -31 & 125317.92 & -602326.3 & 12.18 & 0.28 & B6-7 III-V & -14 & G & \\
\hline $4755-063$ & $\mathrm{I}-29$ & 125256.77 & -601921.9 & 12.18 & 0.33 & B8 V & -32 & G & \\
\hline $4755-064$ & $\mathrm{I}-09$ & 125332.24 & -602219.7 & 12.22 & 0.27 & B6-7 V & -23 & G & \\
\hline $4755-065$ & III-34 & 125355.21 & -602709.8 & 12.25 & 0.18 & B3 V & -22 & G & \\
\hline $4755-066$ & - & 125214.46 & -602549.3 & 12.25 & 0.27 & B3 V & -24 & G & \\
\hline $4755-067$ & IV -30 & 125333.47 & -602420.7 & 12.28 & 0.24 & B5 V & -23 & G & \\
\hline $4755-068$ & $\mathrm{I}-01$ & 125340.76 & -602141.0 & 12.31 & 0.27 & B3 Vn & -20 & G & \\
\hline $4755-069$ & $\mathrm{I}-36$ & 125325.35 & -602021.3 & 12.31 & 0.28 & B5 V & -17 & G & \\
\hline $4755-070$ & II-18 & 125409.06 & -602210.3 & 12.32 & 0.23 & B3 V & -24 & G & \\
\hline $4755-071$ & - & 125333.78 & -602054.3 & 12.35 & 0.32 & B9 III & -24 & G & \\
\hline $4755-072$ & II-16 & 125348.72 & -602039.7 & 12.43 & 0.24 & B5 V & -19 & G & \\
\hline $4755-073$ & $\mathrm{I}-16$ & 125336.13 & -602032.5 & 12.45 & 0.24 & A0 II & -15 & G & \\
\hline $4755-074$ & $\mathrm{I}-20$ & 125318.39 & -602208.1 & 12.47 & 0.29 & B5 III-V & $-36:$ & G & \\
\hline $4755-075$ & $\mathrm{I}-11$ & 125325.87 & -602214.9 & 12.52 & 0.24 & B5 III-V & -22 & G & \\
\hline 4755-076 & $\mathrm{I}-08$ & 125335.60 & -602148.6 & 12.58 & 0.36 & B5 V & -18 & G & \\
\hline $4755-077$ & $\mathrm{I}-37$ & 125337.51 & -601926.8 & 12.59 & 0.32 & B6-7 III & -27 & G & \\
\hline 4755-078 & - & 125455.66 & -602004.3 & 12.60 & 0.32 & B5 Vn & -23 & G & \\
\hline $4755-079$ & I-18 & 125319.54 & -602131.0 & 12.62 & 0.28 & B5 V & -20 & G & \\
\hline $4755-080$ & M & 125318.19 & -601849.8 & 12.62 & 0.35 & B8 III-V & -19 & G & \\
\hline $4755-081$ & - & 125327.77 & -602237.7 & 12.63 & 0.28 & B6-7 IIIn & -18 & G & \\
\hline 4755-082 & IV -25 & 125317.25 & -602252.1 & 12.65 & 0.30 & B8 III-V & -25 & G & \\
\hline $4755-083$ & - & 125507.53 & -602138.0 & 12.68 & 0.34 & B9 III & -16 & G & \\
\hline $4755-084$ & IV-16 & 125340.60 & -602413.0 & 12.75 & 0.25 & B9 III & -15 & G & \\
\hline $4755-085$ & I-34 & 125317.01 & -602031.1 & 12.76 & 0.36 & B9 III & -13 & G & \\
\hline 4755-086 & - & 125245.06 & -601958.5 & 12.79 & 0.39 & A0 III & -35 : & G & \\
\hline $4755-087$ & IV-07 & 125333.93 & -602241.7 & 12.81 & 0.25 & B8 III & -19 & G & \\
\hline $4755-088$ & IV-56 & 125322.35 & -602742.2 & 12.83 & 0.25 & B8 IIIn & -24 & G & \\
\hline 4755-089 & - & 125329.99 & -601451.1 & 12.84 & 0.36 & B8 III & -19 & G & \\
\hline $4755-090$ & III-09 & 125357.13 & -602231.6 & 12.88 & 0.27 & B8 IIIn & -16 & G & \\
\hline 4755-091 & II-34 & 125348.11 & -601745.1 & 12.90 & 0.37 & B8 III & -20 & G & \\
\hline 4755-092 & IV-35 & 125338.09 & -602559.9 & 12.91 & 0.14 & A0 IIp (Si) & -24 & G & \\
\hline $4755-093$ & III-02* & 125344.65 & -602232.4 & 12.91 & 0.28 & B8 III-V & -29 & G & \\
\hline $4755-094$ & IV-45 & 125320.30 & -602535.1 & 12.93 & 0.31 & B8 III & -30 & G & \\
\hline $4755-095$ & III-33 & 125355.12 & -602650.6 & 12.96 & 0.20 & B8 III & -22 & G & \\
\hline $4755-096$ & II-12 & 125350.68 & -602122.2 & 12.96 & 0.29 & B8 III & -13 & G & \\
\hline $4755-097$ & IV $-21^{*}$ & 125334.12 & -602345.6 & 12.96 & 0.34 & A0 III & -20 & G & \\
\hline $4755-098$ & IV-44 & 125322.02 & -602501.2 & 13.00 & 0.25 & B8 IIIn & -20 & G & \\
\hline 4755-099 & - & 125428.30 & -601445.7 & 13.01 & 0.41 & B8 III & -20 & G & \\
\hline $4755-100$ & IV-01 & 125341.77 & -602243.9 & 13.09 & 0.26 & B8 III & -15 & G & \\
\hline
\end{tabular}


C. J. Evans et al.: FLAMES survey of massive stars: MW Clusters, Online Material $p 7$

Table 10. continued.

\begin{tabular}{llccccccll}
\hline \hline ID & Alias & $\alpha(2000)$ & $\delta(2000)$ & $V$ & $B-V$ & Sp. type & $v_{\mathrm{r}}$ & Inst. & Comments \\
\hline $4755-101$ & - & 125252.93 & -601748.8 & 13.10 & 0.28 & B9 II & -22 & G & \\
$4755-102$ & - & 125353.17 & -602124.9 & 13.11 & 0.39 & A2 III & -22 & G & near to II-10 \\
$4755-103$ & I-28 & 125256.56 & -602029.3 & 13.11 & 0.40 & A3 III & -19 & G & \\
$4755-104$ & III-20 & 125403.25 & -602401.5 & 13.13 & 0.27 & A0 IIp (Si) & $-12:$ & G & \\
$4755-105$ & - & 125417.26 & -601754.9 & 13.13 & 0.43 & B9 III & -28 & G & \\
$4755-106$ & - & 125457.47 & -602341.4 & 13.16 & 0.32 & B8 III & -18 & G & \\
$4755-107$ & IV-24 & 125323.56 & -602312.5 & 13.18 & 0.29 & B8 III-V & -20 & G & \\
$4755-108$ & I-21 & 125309.20 & -602203.0 & 13.22 & 0.31 & B8 III-V & -22 & G & \\
\hline
\end{tabular}




\section{J. Evans et al.: FLAMES survey of massive stars: MW Clusters, Online Material $p 8$}

Table 11. NGC 6611: observational Parameters of Target Stars. Cross-identifications in column two are from Walker (W, 1961), Kamp (K, 1974), and Tucholke et al. (T, 1986). WFI photometry is quoted for $V>11.47^{\mathrm{m}}$, brighter than this there were saturation problems and values are from Hillenbrand et al. (1993). Radial velocities $\left(v_{\mathrm{r}}\right)$ are given in $\mathrm{km} \mathrm{s}^{-1}$. Note that stars 6611-001, 6611-005 and 6611-045 are actually outside of the FLAMES field-of-view. Photometry for 6611-040 and 6611-045 is also from Hillenbrand et al. (1993). Instrument codes refer to FEROS (F), Giraffe (G), and ISIS (I).

\begin{tabular}{|c|c|c|c|c|c|c|c|c|c|}
\hline ID & Alias & $\alpha(2000)$ & $\delta(2000)$ & V & $B-V$ & Sp. type & $v_{\mathrm{r}}$ & Inst. & Comments \\
\hline $6611-001$ & W412 & 181858.71 & -135928.1 & 8.18 & 0.34 & B0 III & 86 & $\mathrm{~F}+\mathrm{I}$ & HD168183, MCW660, BD-14 4991 \\
\hline $6611-002$ & W205 & 181836.44 & -134803.1 & 8.18 & 0.43 & $\mathrm{O} 4 \mathrm{~V}((\mathrm{f}+))$ & 14 & $\mathrm{~F}+\mathrm{I}$ & HD168076, MCW656, BD-13ํ4926; H $\alpha=$ wk. P Cyg \\
\hline $6611-003$ & W197 & 181836.06 & -134736.3 & 8.73 & 0.45 & O6-7 V((f))+B0: & 17 & $\mathrm{~F}+\mathrm{I}$ & HD168075, MCW655, BD-134925 \\
\hline $6611-004$ & W401 & 181856.21 & -134831.0 & 8.90 & 0.04 & $08.5 \mathrm{~V}$ & 23 & $\mathrm{~F}+\mathrm{I}$ & HD168137, MCW659, BD-13ํำ 4932 \\
\hline $6611-005$ & - & 182034.10 & -135715.8 & 9.13 & 0.44 & O8 III & 5 & $\mathrm{~F}+\mathrm{I}$ & $\mathrm{HD} 168504, \mathrm{BD}-14^{\circ} 5005$ \\
\hline $6611-006$ & W367 & 181852.70 & -134942.6 & 9.39 & 0.24 & O9.7 IIIp & -1 & $\mathrm{G}+\mathrm{F}+\mathrm{I}$ & $\mathrm{BD}-13^{\circ} 4930$ \\
\hline $6611-007$ & W468 & 181905.58 & -135450.3 & 9.40 & 0.28 & $\mathrm{~B} 0.5 \mathrm{~V}+\mathrm{B} 1:$ & -104 & G & $\mathrm{BD}-13^{\circ} 4934$ \\
\hline $6611-008$ & W246 & 181840.11 & -134518.5 & 9.46 & 0.82 & $\mathrm{O} 7 \mathrm{II}(\mathrm{f})$ & 15 & $\mathrm{G}+\mathrm{I}$ & $\mathrm{BD}-13^{\circ} 4927$ \\
\hline $6611-009$ & W184 & 181834.52 & -135421.9 & 9.73 & 1.35 & K0 V & -42 & G & $\mathrm{BD}-13^{\circ} 4924$ \\
\hline $6611-010$ & W503 & 181911.08 & -135642.8 & 9.75 & 0.49 & B1: e & 4 & $\mathrm{G}$ & BD-134 4936, MWC 918; $\mathrm{H} \alpha=$ broad em. \\
\hline $6611-011$ & W314 & 181845.86 & -134630.8 & 9.85 & 0.58 & O9 V & 17 & $\mathrm{G}+\mathrm{I}$ & $\mathrm{BD}-13^{\circ} 4929$ \\
\hline $6611-012$ & W150 & 181829.98 & -134957.6 & 9.85 & 0.48 & B $0.5 \mathrm{~V}$ & 14 & $\mathrm{G}$ & $\mathrm{BD}-13^{\circ} 4921$ \\
\hline $6611-013$ & W125 & 181826.22 & -135005.5 & 10.01 & 0.47 & $\mathrm{~B} 1 \mathrm{~V}+$ ? & $-92:$ & G & $\mathrm{BD}-13^{\circ} 4920$ \\
\hline $6611-014$ & W175 & 181832.75 & -134511.9 & 10.09 & 0.84 & $\mathrm{O} 5 \mathrm{~V}((\mathrm{f}+))+$ late- $\mathrm{O}$ & 36 & $\mathrm{G}+\mathrm{I}$ & $\mathrm{BD}-13^{\circ} 4923$ \\
\hline $6611-015$ & W280 & 181842.79 & -134650.9 & 10.12 & 0.43 & O9.5 Vn & 23: & $\mathrm{G}+\mathrm{I}$ & $\mathrm{BD}-13^{\circ} 4928$ \\
\hline $6611-016$ & K599 & 181906.48 & -135745.7 & 10.16 & 1.35 & G8 III & -30 & G & faint companion in WFI image \\
\hline $6611-017$ & W166 & 181832.24 & -134848.0 & 10.37 & 0.57 & $09 \mathrm{~V}$ & 19 & $\mathrm{~F}+\mathrm{I}$ & \\
\hline $6611-018$ & W002 & 181802.96 & -134435.0 & 10.56 & 0.35 & B8 III & 16 & $\mathrm{~F}$ & $\mathrm{BD}-13^{\circ} 4914$ \\
\hline 6611-019 & K601 & 181920.01 & -135421.3 & 10.68 & 0.36 & $\mathrm{~B} 1.5 \mathrm{~V}$ & -47 & $\mathrm{~F}$ & BD-13 4937 ; blended in WFI image \\
\hline $6611-020$ & W469 & 181904.89 & -134820.3 & 10.69 & 0.40 & B0.5 Vn & 20 & G & $\mathrm{BD}-13^{\circ} 4933$ \\
\hline $6611-021$ & W254 & 181840.77 & -134652.3 & 10.80 & 0.47 & $\mathrm{~B} 1 \mathrm{~V}$ & -18 & $\mathrm{~F}$ & \\
\hline $6611-022$ & W235 & 181838.84 & -134644.2 & 10.98 & 0.82 & Herbig Be & - & $\mathrm{G}$ & MWC 916; $\mathrm{H} \alpha=$ broad em. \\
\hline $6611-023$ & W483 & 181906.51 & -134330.3 & 10.99 & 0.41 & B3 V & -19 : & G & $\mathrm{BD}-13^{\circ} 4935$ \\
\hline $6611-024$ & W501 & 181909.95 & -135053.0 & 11.19 & 1.67 & $\mathrm{~K} 2: \mathrm{V}$ & -13 & G & \\
\hline $6611-025$ & W223 & 181837.88 & -134635.1 & 11.20 & 0.59 & $\mathrm{~B} 1 \mathrm{~V}$ & 8 & $\mathrm{G}$ & \\
\hline $6611-026$ & W432 & 181900.20 & -135534.3 & 11.25 & 0.53 & F0 III & 0 & G & \\
\hline $6611-027$ & W351 & 181850.81 & -134812.7 & 11.26 & 0.45 & $\mathrm{~B} 1 \mathrm{~V}$ & 0 & $\mathrm{~F}$ & \\
\hline $6611-028$ & W500 & 181909.02 & -134314.8 & 11.28 & 0.43 & B5e & - & $\mathrm{G}$ & $\mathrm{H} \alpha=$ broad em \\
\hline $6611-029$ & W161 & 181830.97 & -134308.2 & 11.29 & 1.05 & $08.5 \mathrm{~V}$ & -6 & $\mathrm{G}+\mathrm{I}$ & \\
\hline 6611-030 & W536 & 181918.49 & -135539.8 & 11.46 & 0.22 & $\mathrm{~B} 1.5 \mathrm{~V}+?$ & 42 & G & \\
\hline $6611-031$ & W407 & 181855.38 & -133910.4 & 11.46 & 0.60 & A3 III & 0 & G & \\
\hline $6611-032$ & W239 & 181840.02 & -135433.4 & 11.48 & 0.36 & $\mathrm{~B} 1.5 \mathrm{~V}$ & 4 & G & \\
\hline $6611-033$ & W210 & 181836.99 & -134752.7 & 11.50 & 0.54 & $\mathrm{~B} 1 \mathrm{~V}$ & 8 & G & \\
\hline 6611-034 & W489 & 181907.33 & -134304.6 & 11.52 & 0.54 & B8 III & -18 & G & \\
\hline 6611-035 & W259 & 181840.98 & -134529.6 & 11.56 & 0.73 & B $0.5 \mathrm{~V}$ & 16 & G & \\
\hline 6611-036 & K592* & 181902.98 & -133604.1 & 11.60 & 0.57 & A3 III & 6 & G & \\
\hline $6611-037$ & K600 & 181916.42 & -135414.1 & 11.62 & 1.38 & $\mathrm{~K} 0 \mathrm{~V}$ & 37 & G & \\
\hline $6611-038$ & W520 & 181913.99 & -135221.5 & 11.66 & 0.45 & B5 IIIn & -15 & G & \\
\hline 6611-039 & W349 & 181850.59 & -134733.9 & 11.69 & 1.35 & G5 III & 5 & G & \\
\hline $6611-040$ & W090 & 181820.20 & -134611.9 & 11.73 & 0.38 & B5 V & 30 & $\mathrm{~F}$ & In WFI chip-gap \\
\hline $6611-041$ & K591 & 181903.23 & -135607.3 & 11.75 & 0.44 & B5 V & 6 & $\mathrm{G}$ & \\
\hline $6611-042$ & W296 & 181844.68 & -134756.3 & 11.81 & 0.49 & B $1.5 \mathrm{~V}$ & 14 & G & \\
\hline $6611-043$ & W117 & 181824.88 & -134242.5 & 11.77 & 1.63 & K0 V & 5 & G & \\
\hline 6611-044 & W417 & 181857.21 & -134137.0 & 11.95 & 1.61 & K0 V & -49 & G & \\
\hline $6611-045$ & W584 & 181823.64 & -133628.2 & 12.02 & 1.05 & O9 V & 6 & $\mathrm{~F}$ & \\
\hline $6611-046$ & K583* & 181829.90 & -133624.8 & 12.04 & 0.42 & B9 III & -1 & G & \\
\hline $6611-047$ & K611* & 181930.03 & -134412.5 & 12.00 & 1.66 & K0 V & -23 & G & \\
\hline $6611-048$ & W290 & 181844.86 & -135622.2 & 12.12 & 0.46 & $\mathrm{~B} 2.5 \mathrm{~V}$ & 8 & G & \\
\hline 6611-049 & W455 & 181902.91 & -134717.5 & 12.12 & 0.54 & A5 II & -15 & G & \\
\hline $6611-050$ & W411 & 181856.97 & -134406.5 & 12.08 & 1.63 & K0 V & 62 & $\mathrm{G}$ & \\
\hline
\end{tabular}


Table 11. continued.

\begin{tabular}{|c|c|c|c|c|c|c|c|c|c|}
\hline ID & Alias & $\alpha(2000)$ & $\delta(2000)$ & $\bar{V}$ & $B-V$ & Sp. type & $v_{\mathrm{r}}$ & Inst. & Comments \\
\hline $6611-051$ & W429 & 181857.38 & -133813.3 & 12.12 & 0.81 & F5 V & 14 & $\mathrm{G}$ & Metal weak? \\
\hline $6611-052$ & W301 & 181845.00 & -134624.8 & 12.19 & 0.58 & $\mathrm{~B} 2 \mathrm{~V}$ & 7 & G & \\
\hline $6611-053$ & W394 & 181856.21 & -135602.0 & 12.19 & 1.18 & G5 III-V & 13 & G & \\
\hline $6611-054$ & K590* & 181850.41 & -135704.3 & 12.27 & 0.28 & A0 II & 48: & G & \\
\hline $6611-055$ & W440 & 181900.55 & -134634.9 & 12.27 & 1.36 & K0 V & 12 & G & \\
\hline $6611-056$ & W484 & 181906.91 & -134504.4 & 12.39 & 0.51 & B8 III & -21 & G & \\
\hline $6611-057$ & W079 & 181818.38 & -134337.2 & 12.44 & 0.63 & A7 II & 2 & G & \\
\hline $6611-058$ & W473 & 181905.73 & -135333.4 & 12.46 & 0.24 & A0 II & -10 & G & \\
\hline $6611-059$ & W346 & 181849.33 & -133924.8 & 12.43 & 1.49 & K0 V & -30 & G & \\
\hline $6611-060$ & T636 & 181922.08 & -134015.5 & 12.51 & 1.45 & A5 II & 50: & G & \\
\hline 6611-061 & K573* & 181806.99 & -134113.3 & 12.54 & 0.77 & F8 V & $-13:$ & G & \\
\hline $6611-062$ & W289 & 181844.11 & -134856.4 & 12.60 & 0.52 & B3 V & 8 & G & \\
\hline $6611-063$ & W444 & 181900.44 & -134240.9 & 12.65 & 0.92 & $\mathrm{~B} 1.5 \mathrm{~V}$ & 7 & G & \\
\hline $6611-064$ & W311 & 181845.60 & -134753.1 & 12.78 & 0.55 & B3 V & 14 & G & \\
\hline $6611-065$ & W504 & 181910.32 & -134903.7 & 12.80 & 0.40 & B9 III & 11 & G & \\
\hline $6611-066$ & W227 & 181838.42 & -134709.0 & 12.83 & 0.62 & B2 V & -50 & G & \\
\hline $6611-067$ & W303 & 181845.88 & -135440.1 & 12.85 & 0.65 & F0 III-V & 30: & G & \\
\hline $6611-068$ & W472 & 181904.72 & -134444.4 & 12.85 & 0.53 & $\mathrm{~B} 3 \mathrm{~V}+$ ? & 2 & G & \\
\hline $6611-069$ & W409 & 181857.39 & -135212.1 & 12.89 & 0.41 & B $2.5 \mathrm{~V}$ & 17 & G & \\
\hline $6611-070$ & W400 & 181855.85 & -134654.0 & 12.88 & 0.59 & B9 III & $-32:$ & G & \\
\hline $6611-071$ & W297 & 181844.54 & -134548.1 & 12.89 & 0.67 & B2 Vn & 1 & G & \\
\hline $6611-072$ & W313 & 181846.15 & -134923.4 & 12.93 & 0.49 & B5 III & 23 & G & \\
\hline $6611-073$ & W135 & 181827.81 & -135504.9 & 12.93 & 0.70 & F0 III-V & 2 & G & \\
\hline $6611-074$ & W272 & 181841.15 & -133736.9 & 12.99 & 0.95 & F5 III-V & $-46:$ & G & \\
\hline $6611-075$ & T639 & 181926.24 & -134725.2 & 13.06 & 0.57 & A7 II & 14 & G & \\
\hline $6611-076$ & W163 & 181830.52 & -133705.6 & 13.05 & 0.80 & F0 III-V & $-34:$ & G & \\
\hline $6611-077$ & W174 & 181833.15 & -135137.1 & 13.05 & 0.90 & F8 V & -10 & G & \\
\hline 6611-078 & W267 & 181841.71 & -134643.8 & 13.11 & 0.52 & B3 V & 5 & G & \\
\hline $6611-079$ & - & 181901.53 & -133557.1 & 13.09 & 0.82 & F0 III & 15 & G & ВКР 29509 \\
\hline $6611-080$ & W222 & 181837.52 & -134339.4 & 13.08 & 1.35 & O7 V((f)) & 16 & G & \\
\hline $6611-081$ & - & 181842.78 & -133557.3 & 13.14 & 0.56 & A7 II & 2 & G & BKP 29510 \\
\hline $6611-082$ & W541 & 181919.13 & -134352.1 & 13.21 & 0.64 & B1-3 V & 2 & G & \\
\hline $6611-083$ & W036 & 181811.15 & -134536.6 & 13.27 & 0.63 & A7 II & 2 & G & \\
\hline 6611-084 & W345 & 181850.28 & -135300.9 & 13.27 & 0.63 & F0 V & 30 & G & \\
\hline $6611-085$ & W336 & 181849.19 & -134804.2 & 13.33 & 0.48 & B5 III & 5 & G & \\
\hline
\end{tabular}

Prefixes in the final column refer to: MWC, (Mount Wilson Catalogue, Merrill \& Burwell 1949); MCW, (Morgan et al. 1955); BKP, (Belikov et al. 1999). Stars marked with "*" are those for which definite visual identifications were not possible from the Kamp (1974) finding chart, with the cross-references taken from Belikov et al. (1999). 
Table 12. Comparison of current classifications with published spectral types. Sources of spectral types are: B54 (Bidelman 1954); BMN99 (Bosch et al. 1999); CL74 (Conti \& Leep 1974); F58 (Feast 1958); F63 (Feast 1963); H60 (Bidelman, from Hernández 1960); HMSM (Hillenbrand et al. 1993); HGS69 (Hiltner et al. 1969); HM69 (Hiltner \& Morgan 1969); H56 (Hoffleit 1956); MCW55 (Morgan et al. 1955); S70 (Schild 1970); T80 (Turner et al. 1980); TrW61 (Trumpler, from Walker 1961); W61 (Walker 1961); W72 (Walborn 1972); W73 (Walborn 1973); W76 (Walborn 1976); and W82 (Walborn 1982).

\begin{tabular}{|c|c|c|c|}
\hline ID & Alias & FLAMES & Published \\
\hline 3293-001 & F004 & B0 Iab & B0 Ib [MCW55]; B0 Ia [H56]; B0 I(b?) [F58]; B0 Ia [W76]; B0 Iab [T80] \\
\hline $3293-002$ & F003 & B0.7 Ib & B0.5 Ib [MCW55]; B0.5 I [H56]; B0.5 Ib [F58]; B0.7 Ia [W76] \\
\hline $3293-003$ & F022 & B1 III & B1 II [F58]; B1 Ib [T80] \\
\hline 3293-004 & F020 & B1 III & B1 III [F58]; B1 II [T80] \\
\hline 3293-005 & F025 & B1 III & B1 III [F58]; B1 II [T80] \\
\hline $3293-006$ & F006 & B1 III & B0.5 III [F58]; B0.5 II [T80] \\
\hline 3293-007 & F008 & B1 III & B0.5 II [H56]; B0.5 III [F58] \\
\hline 3293-008 & F007 & B1 III & B1 III [H56]; B1 III [F58] \\
\hline 3293-009 & F048 & A7 III & A3 [F58] \\
\hline $3293-010$ & F016 & B1 III & B1 III [F58] \\
\hline 3293-011 & F026 & $\mathrm{Be}(\mathrm{B} 1:)$ & B1 III [F58] \\
\hline 3293-012 & F027 & B1 III & B0 II [H56]; B0.5 III [F58] \\
\hline $3293-013$ & F005 & B1 III & B1 III [F58]; B1 IV [T80] \\
\hline 3293-014 & F019 & B0.5 IIIn & B1 III [F58]; B0.5 IIn [T80] \\
\hline $3293-016$ & F023 & $\mathrm{B} 2.5 \mathrm{~V}$ & B1 III [F58] \\
\hline $3293-017$ & F024 & B1 V & B1 III [F58] \\
\hline 3293-018 & F018 & $\mathrm{B} 1 \mathrm{~V}$ & B1 V [F58]; B1 IV [T80] \\
\hline 3293-019 & F014 & B1 V & B1 V [F58]; B1 IV [T80] \\
\hline $3293-020$ & F010 & B1.5 III & B1 V [F58]; B0.5 V [T80] \\
\hline $3293-022$ & F012 & $\mathrm{Be}$ & B1 V [F58]; B1 IVne [T80] \\
\hline 3293-024 & F009 & B1.5 III & B2 V [F58]; B1.5 V [T80] \\
\hline $3293-025$ & F015 & B2 III & B1 V [F58]; B2: IVn [T80] \\
\hline $3293-026$ & F013 & B2 III & B2 V [F58]; B1 V [T80] \\
\hline 3293-029 & F028 & B0.5-1.5 Vn & B1 V [F58] \\
\hline $3293-030$ & F017 & $\mathrm{B} 2 \mathrm{~V}$ & B2-3 V: [F58] \\
\hline 3293-031 & F033 & $\mathrm{B} 2 \mathrm{~V}$ & B2 V [F58] \\
\hline $3293-032$ & F029 & B0.5-1.5 Vn & B2 V [F58] \\
\hline $3293-033$ & F041 & B8 III & B8 [F58] \\
\hline $3293-035$ & F045 & $\mathrm{B} 2 \mathrm{~V}$ & B8 [F58] \\
\hline $3293-036$ & F036 & A5 III & A2 [F58] \\
\hline 3293-039 & F051 & A3 III & A0 [F58] \\
\hline $3293-042$ & F044 & A3 III & B [F58] \\
\hline 3293-044 & F039 & A3 III & A [F58] \\
\hline $3293-046$ & F046 & A7 III & A0 [F58] \\
\hline $4755-001$ & A & B9 Ia & B9 Ia [B54]; B9 Ia-Iab [H60]; B9 Iab [F63]; B9 Ia [S70] \\
\hline
\end{tabular}


Table 12. continued.

\begin{tabular}{|c|c|c|c|}
\hline ID & Alias & FLAMES & Published \\
\hline $4755-002$ & B & B3 Ia & B3 Iab [B54]; B3 Iab [H60]; B3 Ia [F63]; B5 Ia [HGS69]; B5 Ia [S70]; B3 Ia [W72] \\
\hline 4755-003 & $\mathrm{C}$ & B2 III & B3 Ib [H60]; B2 Ib [F63]; B2 Ib [S70] \\
\hline 4755-004 & $\mathrm{I}-06$ & $\mathrm{~B} 1.5 \mathrm{Ib}$ & B2 Ib: [H60]; B1.5 Ib [F63]; B1.5 Ib [S70] \\
\hline 4755-005 & II-23 & B2 III & B0 V? [F63], Feast noted star as a double-lined binary; B0.5 Vn [S70] \\
\hline $4755-006$ & $\mathrm{E}$ & B1 III & B1 III [F63]; B1 III [S70] \\
\hline 4755-007 & III-05 & B1 V & B2:: [H60]; B0.5 V [F63]; B0.5 IVn [S70] \\
\hline $4755-008$ & $\mathrm{I}-05$ & $\mathrm{~B} 0.5 \mathrm{~V}$ & B1-2 II-II [H60]; B0.5 III[n] [F63] \\
\hline 4755-009 & $\mathrm{F}$ & $\mathrm{B} 1 \mathrm{~V}$ & B0.5-1 II-III [H60]; B2 III [F63]; B2 III [S70] \\
\hline $4755-010$ & II-01 & B1 V & B1 V [F63]; B0.5 V [S70] \\
\hline 4755-011 & $\mathrm{R}$ & $\mathrm{B} 1.5 \mathrm{~V}$ & B1 V [F63]; B2 IV [S70] \\
\hline 4755-012 & III-07 & $\mathrm{B} 1.5 \mathrm{~V}$ & B1 V [F63]; B1.5 V [S70] \\
\hline 4755-013 & IV-18 & $\mathrm{B} 1.5 \mathrm{~V}$ & B2: V [F63] \\
\hline 4755-014 & S & $\mathrm{Be}(\mathrm{B} 1:)$ & Be [F63]; B1.5 Vpne [S70] \\
\hline $4755-015$ & G & B1 V & B0.5 V [F63] \\
\hline 4755-016 & III-01 & $\mathrm{B} 1.5 \mathrm{~V}$ & B1 V [F63] \\
\hline 4755-018 & IV-17 & Be (B1.5:) & Bnne [F63]; B2 IVne [S70] \\
\hline 4755-021 & I & B1.5 Vn & B1 Vn: [F63] \\
\hline 4755-022 & $\mathrm{I}-17$ & B2.5 Vn & Bnn [F63] \\
\hline $4755-023$ & $\mathrm{~J}$ & B2 V & B1: V: [F63] \\
\hline 4755-034 & $\mathrm{K}$ & B3 V & B8 V [F63] \\
\hline $4755-080$ & $\mathrm{M}$ & B8 III-V & $\mathrm{A} 0: \mathrm{V}[\mathrm{F} 63]$ \\
\hline $6611-001$ & W412 & B0 III & B0 III [MCW55]; O9.5 I [HMSM]; O9.5 I [BMN99] \\
\hline $6611-002$ & W205 & $\mathrm{O} 4 \mathrm{~V}\left(\left(\mathrm{f}^{(+)}\right)\right)$ & O5 [MCW55]; O6 [TrW61]; O5 [HM69]; O4 V ((f)) [W73]; O4 ((f)) [CL74]; O5 V((f*)) [HMSM]; O4 V((f+)) [BMN99] \\
\hline $6611-003$ & W197 & O6-7 V((f))+B0: & O7 [MCW55]; O8 [TrW61]; O7 [HM69]; O6.5 III((f)) [CL74]; O6 V((f)) [W82]; O7 V((f)) [HMSM]; O6-7 V((f)) [BMN99] \\
\hline $6611-004$ & W401 & $08.5 \mathrm{~V}$ & O8 V [MCW55]; O8 [TrW61]; O8 V [HM69]; O8.5 V [HMSM]; O8.5 V [BMN99] \\
\hline $6611-005$ & HD168504 & O8 III & O8 III [CL74]; O7.5 III((f)) [HMSM] \\
\hline $6611-006$ & W367 & O9.7 IIIp & В0 [TrW61]; O9.5 V [HM69]; O9.5 V [HMSM]; O9.5 V [BMN99] \\
\hline $6611-007$ & W468 & B0.5 V + B1: & B1 V [W61]; B1 Vp [HMSM] \\
\hline 6611-008 & W246 & $\mathrm{O} 7 \mathrm{II}(\mathrm{f})$ & O9 [TrW61]; O8f [HM69]; O7 Ib(f) [W82]; O7 II(f) [HMSM]; O7 II(f) [BMN99] \\
\hline 6611-009 & W184 & K0 V & K2 III [W61] \\
\hline $6611-010$ & W503 & B1: e & B0-5e [W61]; Herbig Be [HMSM] \\
\hline $6611-011$ & W314 & $\mathrm{O} 9 \mathrm{~V}$ & B0nn [TrW61]; O9.5 V [HM69]; B0 V [HMSM]; B0 V [BMN99] \\
\hline $6611-012$ & W150 & B $0.5 \mathrm{~V}$ & O9 V [W61]; B0.5 V [HM69]; B0.5 V [HMSM] \\
\hline $6611-013$ & W125 & $\mathrm{B} 1 \mathrm{~V}+?$ & B0-1 V [W61]; B1.5 V [HM69]; B1 V [HMSM] \\
\hline $6611-014$ & W175 & $\mathrm{O} 5 \mathrm{~V}\left(\left(\mathrm{f}^{(+)}\right)\right)+$late- $\mathrm{O}$ & O6 [HM69]; O5.5 V((f)) [HMSM]; O5.5 V((f)) [BMN99] \\
\hline $6611-015$ & W280 & O9.5 Vn & B0nn [TrW61]; O9.5 V [HMSM] \\
\hline $6611-016$ & K599 & G8 III & late-G [BMN99] \\
\hline $6611-017$ & W166 & $\mathrm{O} 9 \mathrm{~V}$ & O9 [TrW61]; O9 V [HM69]; O8.5 V [HMSM]; O8.5 V [BMN99] \\
\hline $6611-018$ & W002 & B8 III & B8 V [W61] \\
\hline $6611-019$ & K601 & $\mathrm{B} 1.5 \mathrm{~V}$ & B1.5 V [HMSM] \\
\hline
\end{tabular}


Table 12. continued.

\begin{tabular}{|c|c|c|c|}
\hline ID & Alias & FLAMES & Published \\
\hline $6611-020$ & W469 & B0.5 Vn & B0-5e [W61]; B1.5 V [HM69]; B0.5 Ve [HMSM] \\
\hline $6611-021$ & W254 & B1 V & B2 [TrW61]; B1 V [HMSM]; B0-2 V [BMN99] \\
\hline $6611-022$ & W235 & Herbig Be & Herbig Be [HMSM] \\
\hline $6611-025$ & W223 & B1 V & B1 V [HM69]; B1 V [HMSM] \\
\hline $6611-027$ & W351 & $\mathrm{B} 1 \mathrm{~V}$ & B1e [TrW61]; B1 Vne [HM69]; B1 V [HMSM] \\
\hline $6611-028$ & W500 & B5 IIIe & B2 V (e?) [BMN99] \\
\hline $6611-029$ & W161 & $08.5 \mathrm{~V}$ & O8.5 V [HMSM] \\
\hline $6611-030$ & W536 & $\mathrm{B} 1.5 \mathrm{~V}+?$ & B $1.5 \mathrm{~V}$ [HMSM] \\
\hline $6611-032$ & W239 & $\mathrm{B} 1.5 \mathrm{~V}$ & B1 V [HMSM] \\
\hline $6611-033$ & W210 & B1 V & B3?e V [W61]; B1.5 V [HM69]; B1 III [HMSM] \\
\hline $6611-034$ & W489 & B8 III & B8 V [BMN99] \\
\hline $6611-035$ & W259 & B $0.5 \mathrm{~V}$ & B0.5 V [HMSM] \\
\hline $6611-038$ & W520 & B5 IIIn & B7: V [BMN99] \\
\hline $6611-040$ & W090 & B5 V & B3-5 V [BMN99] \\
\hline $6611-042$ & W296 & $\mathrm{B} 1.5 \mathrm{~V}$ & B2 V [HM69]; B1.5 V [HMSM] \\
\hline $6611-045$ & W584 & O9 V & O9 V [HMSM] \\
\hline $6611-048$ & W290 & $\mathrm{B} 2.5 \mathrm{~V}$ & B1 V [BMN99] \\
\hline $6611-050$ & W411 & K0 V & G-K [BMN99] \\
\hline $6611-052$ & W301 & B2 V & B2 V [HMSM] \\
\hline $6611-056$ & W484 & B8 III & B5-6 V [BMN99] \\
\hline $6611-062$ & W289 & B3 V & B0-2 V [BMN99] \\
\hline $6611-063$ & W444 & $\mathrm{B} 1.5 \mathrm{~V}$ & B1.5 V [HMSM] \\
\hline $6611-064$ & W311 & B3 V & B2.5 Ve [HMSM] \\
\hline $6611-065$ & W504 & B9 III & B9: V [BMN99] \\
\hline $6611-066$ & W227 & $\mathrm{B} 2 \mathrm{~V}$ & B1.5 Ve [HMSM] \\
\hline $6611-068$ & W472 & $\mathrm{B} 3 \mathrm{~V}+$ ? & B3 Ve [HMSM] \\
\hline $6611-069$ & W409 & $\mathrm{B} 2.5 \mathrm{~V}$ & B2 V [BMN99] \\
\hline $6611-070$ & W400 & B9 III & B9 V [BMN99] \\
\hline $6611-071$ & W297 & B2 V & B1.5: V [HMSM] \\
\hline $6611-072$ & W313 & B5 III & B9: V [BMN99] \\
\hline $6611-076$ & W163 & F0 III-V & F7 [BMN99] \\
\hline $6611-077$ & W174 & F8 V & F-G [BMN99] \\
\hline $6611-080$ & W222 & $\mathrm{O} 7 \mathrm{~V}((\mathrm{f}))$ & O7 III((f)) [HMSM] \\
\hline $6611-082$ & W541 & B1-3 V & B2.5 V [HMSM] \\
\hline $6611-085$ & W336 & B5 III & B5 V [BMN99] \\
\hline
\end{tabular}

